\title{
A remarkable DGmodule model for configuration spaces
}

\author{
PASCAL LAMBRECHTS \\ DON STANLEY
}

\begin{abstract}
Let $M$ be a simply connected closed manifold and consider the (ordered) configuration space $F(M, k)$ of $k$ points in $M$. In this paper we construct a commutative differential graded algebra which is a potential candidate for a model of the rational homotopy type of $F(M, k)$. We prove that our model it is at least a $\Sigma_{k}$-equivariant differential graded model.
\end{abstract}

We also study Lefschetz duality at the level of cochains and describe equivariant models of the complement of a union of polyhedra in a closed manifold.

55P62, 55R 80

\section{Introduction}

Let $M$ be a closed simply connected triangulable manifold of dimension $m$. The (ordered) configuration space of $k$ points in $M$ is the space

$$
F(M, k):=\left\{\left(x_{1}, \cdots, x_{k}\right) \in M^{k}: x_{i} \neq x_{j} \text { for } i \neq j\right\} .
$$

An interesting problem is whether the homotopy type of that configuration space depends only on the homotopy type of $M$. Longoni and Salvatore [15] have discovered an example of two homotopy equivalent manifolds whose configuration spaces of two points are not homotopy equivalent. Their examples are not simply connected. By contrast, a general position argument implies that for a 2-connected closed manifold the configuration space of two points depends only on the homotopy type of the manifold. More generally we have proved in [13] that the rational homotopy type of $F(M, 2)$ depends only on the rational homotopy type of $M$, under the 2-connectivity hypothesis, and we have build an explicit model (in the sense of Sullivan) of that configuration space out of a model of $M$.

The goal of the present paper is to exhibit a promising candidate for the model of the rational homotopy type of the $F(M, k)$. To explain this, first recall the Sullivan functor

$$
A_{\mathrm{PL}}: \text { Top } \rightarrow \text { CDGA }
$$


where CDGA is the category of commutative differential graded algebras. The main feature of this functor is that the rational homotopy type of a simply connected space of finite type, $X$, is encoded in any CDGA quasi-isomorphic to $A_{\mathrm{PL}}(X)$. Such a CDGA is called a $C D G A-$ model of $X$.

In [12] we have proved that any simply connected manifold $M$ admits a CDGA model, $(A, d)$, such that $A$ is a Poincaré duality algebra of dimension $m=\operatorname{dim} M$. We can then define a diagonal class

$$
\Delta:=\sum_{\lambda}(-1)^{\operatorname{deg}\left(a_{\lambda}\right)} a_{\lambda} \otimes a_{\lambda}^{*} \in A \otimes A
$$

where $\left\{a_{\lambda}\right\}$ is a basis of $A$ and $\left\{a_{\lambda}^{*}\right\}$ is the Poincare dual basis. In the present paper we describe a CDGA

$$
F(A, k):=\left(\frac{A^{\otimes k} \otimes \mathrm{E}\left(g_{i j}: 1 \leq i<j \leq k\right)}{\text { (Arnold and symmetry relations) }}, d\left(g_{i j}\right)=\pi_{i j}^{*}(\Delta)\right)
$$

where $\mathrm{E}\left(g_{i j}\right)$ is an exterior algebra on $\left(\begin{array}{l}k \\ 2\end{array}\right)$ generators $g_{i j}$ of degree $m-1, \pi_{i}^{*}(a)=$ $1^{\otimes i-1} \otimes a \otimes 1^{\otimes k-i} \in A^{\otimes k}$ and $\pi_{i j}^{*}(a \otimes b)=\pi_{i}^{*}(a) \cdot \pi_{j}^{*}(b)$ (see Definition 3.4 for a complete definition.)

When $k=2, F(A, 2)$ is weakly equivalent to the CDGA-model of $F(M, 2)$ built in [13, Theorem 5.6], and when $M$ is a complex projective variety then $F\left(H^{*}(M ; \mathbb{Q}) ; k\right)$ is equivalent to the Fulton-MacPherson-Kriz CDGA-model of $F(M, k)$ built in [8] and [11]. We are not able to prove in general that for $k \geq 3, F(A, k)$ is a CDGA-model of $F(M, k)$ but at least we can prove that it is an equivariant DGmodule model of it. More precisely the inclusion $F(M, k) \hookrightarrow M^{k}$ and Kunneth quasi-isomorphism induce an $A_{\mathrm{PL}}(M)^{\otimes k}$-module structure on $A_{\mathrm{PL}}(F(M, k))$. Suppose given quasiisomorphisms of CDGA, $A \stackrel{\simeq}{\cong} \stackrel{\simeq}{\rightrightarrows} A_{\mathrm{PL}}(M)$. Our main result (Theorem 10.1) states that $A_{\mathrm{PL}}(F(M, k))$ and $F(A, k)$ are weakly equivalent $R^{\otimes k}$-DGmodules, even $\Sigma_{k}$ equivariantly where $\Sigma_{k}$ is the symmetric group on $k$ letters acting by permutation of the factors.

Our proof goes through an "equivariant cochain-level Lefschetz duality theorem for a system of subpolyhedra in a closed manifold." In more detail, classical Lefschetz duality determines $H^{*}(W \backslash X)$ from the map $H_{*}(X) \rightarrow H_{*}(W)$ when $X$ is a subpolyhedron of a closed oriented manifold $W$. In [14] we studied Lefschetz duality at the level of models instead of homology. In this paper we generalize this further by considering $X$ as a union of a finite family of subpolyhedra $\left\{X_{e} \hookrightarrow W\right\}_{e \in E}$. The idea is that Lefschetz duality gives a weak equivalence between $C^{*}\left(W \backslash \bigcup_{e \in E} X_{e}\right)$ and the mapping cone of the dual of the map $C^{*}(W) \rightarrow C^{*}\left(\cup_{e \in E} X_{e}\right)$. On the other hand a generalized 
Mayer-Vietoris theorem gives a weak equivalence between $C^{*}\left(\cup_{e \in E} X_{e}\right)$ and a chain complex built out of the chain complexes $C^{*}\left(\bigcap_{e \in \gamma} X_{e}\right)$ for nonempty subsets $\gamma \subset E$. When a discrete group $G$ acts on the manifold $W$ preserving in a certain sense the system $\left\{X_{e} \hookrightarrow W\right\}_{e \in E}$, all these weak equivalences can be chosen to be equivariant.

This generalized Lefschetz duality can be applied to the system of partial diagonals $\Delta_{i j}=\left\{\left(x_{1}, \cdots, x_{k}\right) \in M^{k}: x_{i}=x_{j}\right\}$ so that $F(M, k)=M^{k} \backslash \bigcup_{1 \leq i<j \leq k} \Delta_{i j}$. This approach was already taken by Bendersky and Gitler in [2]. The difference with their paper is that we apply Lefschetz duality at the level of models in order to get a model of $F(M, k)$ instead of a model of the pair $\left(M^{k}, \bigcup_{1 \leq i<j \leq k} \Delta_{i j}\right)$ as they do. Also we carefully study the action of the symmetric group on that model.

The model (1-1) also gives rise to a spectral sequence by filtering by the length in the variables $g_{i j}$. This spectral sequence coincides with the two spectral sequences studied by Félix and Thomas [7]. In particular, as Félix and Thomas show in that paper, this spectral sequence does not always collapse when $k \geq 4$. Also the fixed point CDGA, $F(A, k)^{\Sigma_{k}}$, is a DGmodule model of the unordered configuration space, and Félix and Tanré proved in [6] that the associated spectral sequence does collapse.

In the Section 11 we explain how our approach could be useful to the study of other complement spaces, such as complements of unions of projective subspaces in $\mathbb{C} P(n)$.

Here is a plan of this paper. In Section 2 we recall some notation in particular for the suspension and dual of DGmodules and for their mapping cones. In Section 3 we construct in detail the CDGA $F(A, k)$ associated to a Poincaré duality CDGA $A$. In Section 4 we introduce a variant $\widehat{C}^{*}$ of the cochain algebra functor $C^{*}$ for which the excision quasi-isomorphism is actually an isomorphism. In Section 5 we fix some notation for the action of a discrete group on DGmodules. In Section 6 we establish an equivariant cochain level Lefschetz duality theorem giving a model of the complement $W \backslash X$. In Section 7 we study, for a set of subpolyhedra $\left\{X_{e} \subset W\right\}_{e \in E}$, cubical diagrams like $\{$ subsets $\gamma \subset E\} \rightarrow\left\{C^{*}\left(\bigcap_{e \in \gamma} X_{e}\right)\right\}$ and define their total cofibres which will turn out to be models for the cochains on the complement of the union of polyhedra, $C^{*}\left(W \backslash \bigcup_{e \in E} X_{e}\right)$. In Section 8 we put an action on the cubical diagrams and total cofibres from the previous section. In Section 9 we finally establish the equivariant cochain level Lefschetz duality for a system of polyhedra (Theorem 9.2). In Section 10 we apply the above theory to prove that $F(A, k)$ is an equivariant DGmodule model of $F(M, k)$. The Section 11 is an informal discussion about other possible applications of this approach.

Acknowledgments We thank Soren Illmann for discussions on simplicial actions. We acknowledge support of the institute Mittag-Leffler where part of this research was done during a common stay of the two authors. 


\section{Notation}

In this short section we recall some standard notation.

We fix a ground field $\mathbf{k}$. We will consider noncommutative and commutative nonnegatively graded differential algebras, or DGA and CDGA for short. The degrees are written as superscripts and the differential increases the degree. If $R$ is a (C)DGA we will consider also right differential graded modules over $R$ ( $R$-DGmodules for short see Félix, Halperin and Thomas [5] for the precise definitions).

The $k$-th suspension of an $R$-DGmodule $M$ is the $R$-DGmodule $s^{k} M$ defined by

- $\left(s^{k} M\right)^{i}=M^{i+k}$ as vector spaces;

- $\left(s^{k} x\right) \cdot r=s^{k}(x \cdot r)$ for $x \in M, r \in R$;

- $d\left(s^{k} x\right)=(-1)^{k} s^{k}(d x)$ for $x \in M$.

Therefore $\operatorname{deg}\left(s^{k} x\right)=-k+\operatorname{deg}(x)$. We have a natural isomorphism $s^{k} M \otimes s^{l} N \cong$ $s^{k+l}(M \otimes N)$ sending $s^{k} x \otimes s^{l} y$ to $(-1)^{l \operatorname{deg}(x)} s^{k+l}(x \otimes y)$.

The dual of a graded vector space $V$ is the graded vector space $\# V$ defined by

$$
(\# V)^{k}=\operatorname{Hom}\left(V^{-k}, \mathbf{k}\right) .
$$

If $M$ is a right $R$-DGmodule then $\# M$ inherits an obvious left $R$-DGmodule structure. When $R$ is a commutative DGA, we can turn \# $M$ into a right $R$-DGmodule structure by the rule

$$
\phi \cdot r:=(-1)^{\operatorname{deg}(\phi) \cdot \operatorname{deg}(r)} r \cdot \phi, \text { for } r \in R, \phi \in \# M .
$$

We have canonical isomorphisms $s^{k} \# M \cong \# s^{k} M$ for all $k$ given by $\left(s^{k} f\right)\left(s^{k} x\right)=$ $(-1)^{k \operatorname{deg}(f)} f(x)$, and, under a finite type assumption, $\# M \otimes \# N \cong \#(M \otimes N)$, given by $(f \otimes g)(x \otimes y)=(-1)^{\operatorname{deg}(g) \cdot \operatorname{deg}(x)} f(x) \cdot g(y)$.

If $f:\left(M, d_{M}\right) \rightarrow\left(N, d_{N}\right)$ is a morphism of $R$-DGmodule, the mapping cone of $f$ is the $R$-DGmodule

$$
C(f):=\left(N \oplus_{f} s M, d\right)
$$

defined by

- $C(f)=N \oplus s M$ as $R$-module;

- $d(y, s x)=\left(d_{N}(y)+f(x),-s\left(d_{M}(x)\right)\right)$ for $x \in M, y \in N$. 


\section{The CDGA $F(A, k)$}

An oriented Poincaré algebra of formal dimension $m$ is a couple $(A, \omega)$ where $A$ is a graded commutative $\mathbf{k}$-algebra and $\omega: A^{m} \rightarrow \mathbf{k}$ is a linear form such that each pairing $A^{i} \otimes A^{m-i} \rightarrow \mathbf{k}, a \otimes b \mapsto \omega(a . b)$ is nondegenerate. When $A$ is also equipped with a differential that makes it a CDGA, the following definition, which comes from [13, Definition 4.6] or [12, Definition 2.2], expresses the compatibility between the Poincaré duality and the CDGA structures:

Definition 3.1 An oriented differential Poincaré duality algebra or oriented Poincaré duality $C D G A$ is a triple $(A, d, \omega)$ such that

(i) $(A, d)$ is a CDGA;

(ii) $(A, \omega)$ is an oriented Poincaré duality algebra of formal dimension $m$;

(iii) $\omega(d A)=0$.

Note that when $(A, \omega)$ is a connected Poincare algebra and $(A, d)$ is a CDGA such that the class of maximal degree represents a nontrivial homology class then $(A, d, \omega)$ is a Poincaré duality CDGA, as proved in [13, Proposition 4.8]. The main result of [12] states that any closed oriented simply connected manifold admits a CDGA-model which is a connected Poincaré duality CDGA.

Let $A$ be an oriented Poincaré duality CDGA of formal dimension $m$. Next we recall the diagonal class $\Delta \in(A \otimes A)^{m}$ as defined in [13, Definition 4.4]. Let $\left\{a_{\lambda}\right\}_{0 \leq \lambda \leq N}$ be a basis of $A$ and $\left\{a_{\lambda}^{*}\right\}$ be its Poincare dual basis with respect to the orientation, that is $\omega\left(a_{\lambda} \cdot a_{\mu}^{*}\right)=\delta_{\lambda \mu}$ where $\delta_{\lambda \mu}$ is the Kronecker symbol. The diagonal class is

$$
\Delta:=\sum_{\lambda}(-1)^{\operatorname{deg}\left(a_{\lambda}\right)} a_{\lambda} \otimes a_{\lambda}^{*} \in A \otimes A .
$$

It is proved in [13, Proposition 4.3, Proposition 4.11, and remark after Definition 4.4] that $\Delta$ is a cocycle of degree $m$ which is independent of the choice of the basis. When $A$ is connected this diagonal class is also, up to a scalar multiple, independent of the choice of the orientation. See also Abrams [1], where it is explained how a Poincaré duality algebra, as a Frobenius algebra, becomes a coalgebra, and hence the diagonal class can also be seen as the coproduct of the orientation class.

Consider the CDGA $A^{\otimes k}=A \otimes \cdots \otimes A$. For $1 \leq i \leq k$ we consider the CDGA maps

$$
\pi_{i}^{*}: A \rightarrow A^{\otimes k}, a \mapsto \underbrace{1 \otimes \cdots \otimes 1}_{i-1} \otimes a \otimes \underbrace{1 \otimes \cdots \otimes 1}_{k-i}
$$

Algebraic 83 Geometric Topology, Volume 8 (2008) 
and for $1 \leq i<j \leq k$ the maps

$$
\pi_{i j}^{*}: A \otimes A \rightarrow A^{\otimes k}, a \otimes b \mapsto \underbrace{1 \otimes \cdots \otimes 1}_{i-1} \otimes a \otimes \underbrace{1 \otimes \cdots \otimes 1}_{j-i-1} \otimes b \otimes \underbrace{1 \otimes \cdots \otimes 1}_{k-j},
$$

that is $\pi_{i j}^{*}(a \otimes b)=\pi_{i}^{*}(a) \cdot \pi_{j}^{*}(b)$.

Consider the relative Sullivan algebra [5, Section 14]

$$
\left(A^{\otimes k} \otimes \wedge\left(g_{i j}: 1 \leq i<j \leq k\right), d\right)
$$

with $\operatorname{deg}\left(g_{i j}\right)=m-1$ and $d\left(g_{i j}\right)=\pi_{i j}^{*}(\Delta)$. Notice that $d^{2}=0$ because $\Delta$ is a cocycle. By convention we set

$$
g_{j i}=(-1)^{m} g_{i j} .
$$

Let $I$ be the ideal of $A^{\otimes k} \otimes \wedge\left(g_{i j}\right)$ generated by the following relations:

(i) the Arnold or three-terms relations

$$
g_{i j} g_{j l}+g_{j l} g_{l i}+g_{l i} g_{i j} \quad \text { for } 1 \leq i<j<l \leq k ;
$$

(ii) the symmetry relations

$$
\left(\pi_{i}^{*}(a)-\pi_{j}^{*}(a)\right) g_{i j} \quad \text { for } 1 \leq i<j \leq k \text { and } a \in A ;
$$

(iii) $g_{i j}^{2}=0 \quad$ for $1 \leq i<j \leq k$.

Lemma 3.2 The ideal I generated by relations (i)-(iii) above is a differential ideal of $\left(A^{\otimes k} \otimes \wedge\left(g_{i j}\right), d\right)$.

Proof One computes that

$$
\begin{aligned}
\left(d\left(g_{i j}-g_{i l}\right)\right) \cdot g_{j l} & =\left(\pi_{i j}^{*}(\Delta)-\pi_{i l}^{*}(\Delta)\right) \cdot g_{j l} \\
& =\sum_{\lambda} \pi_{i}^{*}\left(a_{\lambda}\right)\left(\pi_{j}^{*}\left(a_{\lambda}^{*}\right)-\pi_{l}^{*}\left(a_{\lambda}^{*}\right)\right) g_{j l} \in I
\end{aligned}
$$

and this easily implies that $d\left(g_{i j} g_{j l}+g_{j l} g_{l i}+g_{l i} g_{i j}\right) \in I$.

Using Poincaré duality, it is straightforward to check that $(1 \otimes a) \cdot \Delta-(a \otimes 1) \cdot \Delta=0$ in $A \otimes A$ (see [13, Lemma 4.5]). This implies that $d\left(\left(\pi_{i}^{*}(a)-\pi_{j}^{*}(a)\right) g_{i j}\right) \in I$.

It remains to prove that $d\left(g_{i j}^{2}\right) \in I$. If $m$ is even it is immediate by the Leibniz rule. If $m$ is odd we can choose a basis $\left\{a_{\lambda}\right\}_{0 \leq \lambda \leq N}$ of $A$ such that $a_{\lambda}^{*}=a_{N-\lambda}$, hence $\Delta=\sum_{\lambda=0}^{(N-1) / 2}(-1)^{\left|a_{\lambda}\right|}\left(a_{\lambda} \otimes a_{N-\lambda}-a_{N-\lambda} \otimes a_{\lambda}\right)$. From the symmetry relations (ii) one deduces easily that for $a, b \in A$ we have $\pi_{i j}^{*}\left(a \otimes b-(-1)^{|a||b|} b \otimes a\right) g_{i j} \in I$. Therefore $d\left(g_{i j}^{2}\right)=2 \pi_{i j}^{*}(\Delta) g_{i j} \in I$. 
Remark 3.3 The hypothesis that $A$ is a Poincaré duality CDGA is essential for making $I$ a differential ideal, hence for $F(A, k)$ below to be a CDGA.

Definition 3.4 Let $(A, d)$ be a Poincaré duality CDGA of formal dimension $m$. We define the $k$-configuration $C D G A$ as

$$
F(A, k):=\left(\frac{A^{\otimes k} \otimes \wedge\left(g_{i j}: 1 \leq i<j \leq k\right)}{I}, d\left(g_{i j}\right)=\pi_{i j}^{*}(\Delta)\right)
$$

where $g_{i j}, \Delta, \pi_{i j}^{*}$, and $I$ are defined as above. We equip this CDGA with a left action of the symmetric group $\Sigma_{k}$ on $k$ letters generated by

(i) $\sigma \cdot\left(\pi_{i}^{*}(a)\right)=\pi_{\sigma(i)}^{*}(a) \quad$ for $1 \leq i \leq k, a \in A$, and $\sigma \in \Sigma_{k}$;

(ii) $\sigma \cdot g_{i j}=g_{\sigma(i) \sigma(j)} \quad$ for $1 \leq i<j \leq k$ and $\sigma \in \Sigma_{k}$.

When $A$ is connected, since the diagonal class is independent of the orientation (up to a scalar multiple), the CDGA $F(A, k)$ does not depend on the choice of the orientation.

\section{The cochain functor $C^{*}$ and excision isomorphisms}

In this paper we will consider mainly the two following contravariant cochain functors:

- the singular cochain functor with coefficients in a field $\mathbf{k}$,

$$
S^{*}(-; \mathbf{k}): \text { Top } \rightarrow \mathbf{k}-\mathrm{DGA}
$$

where the algebra structure comes from the cup product defined through the usual front face/back face formula;

- the Sullivan functor of piecewise polynomial forms with coefficients in a field $\mathbf{k}$ of characteristic zero,

$$
A_{\mathrm{PL}}(-; \mathbf{k}): \text { Top } \rightarrow \mathbf{k}-\mathrm{CDGA}
$$

as defined in [3] or [5, Section 10].

We will denote by $C^{*}$ either of the two functors $S^{*}(-; \mathbf{k})$ or $A_{\mathrm{PL}}(-; \mathbf{k})$. Notice that an element $\omega \in C^{*}(X)$ is completely determined by its values $\langle\omega, \sigma\rangle$ (which belong to $\mathbf{k}$ when $C^{*}=S^{*}(-; \mathbf{k})$ and to the CDGA $\left(A_{\mathrm{PL}}^{*}\right)_{\operatorname{deg}(\sigma)}$ defined in $[5$, Section 10 (c)] when $\left.C^{*}=A_{\mathrm{PL}}\right)$ on singular simplices $\sigma$ in the singular simplicial set $S_{\bullet}(X)$.

The functor $C^{*}$ extends to pairs of topological spaces by $C^{*}(X, A):=\operatorname{ker}\left(C^{*}(X) \rightarrow\right.$ $\left.C^{*}(A)\right)$. If $(X, A)$ is a pair of topological spaces and if $i:\left(X^{\prime}, A^{\prime}\right) \subset(X, A)$ is a 
subpair such that $\overline{X \backslash X^{\prime}} \subset \operatorname{int}(A)$ and $A^{\prime}=X^{\prime} \cap A$ then the excision theorem implies that the restriction map

$$
C^{*}(i): C^{*}(X, A) \stackrel{\simeq}{\rightarrow} C^{*}\left(X^{\prime}, A^{\prime}\right)
$$

is a quasi-isomorphism. However $C^{*}(i)$ is almost never an isomorphism. We show now how we can replace $C^{*}$ by a quasi-isomorphic functor such that the morphism induced by $i$ is indeed an isomorphism, at least on suitable triangulated pairs. This will be useful in our proof of Theorem 6.3 of equivariant cochain level Lefschetz duality.

Let $\mathcal{K}$ be the category of ordered simplicial complexes defined as follows. An (abstract) simplicial complex is a collection of finite nonempty sets, called simplices, such that every nonempty subset of a simplex is also a simplex [16, Section 3]. The union of that collection is the set of vertices. An object of $\mathcal{K}$ is a simplicial complex with a partial order on the vertices such that each simplex is linearly ordered. A morphism of $\mathcal{K}$ is a simplicial map that preserves the order of the vertices. We denote by $|K|$ the geometric realization of an ordered simplicial complex $K$.

Our goal is to build a functor

$$
\widehat{C}^{*}: \mathcal{K} \rightarrow \mathbf{k}-(\mathrm{C}) \mathrm{DGA}
$$

satisfying the two following properties:

(A) There is a natural quasi-isomorphism of (C)DGA $C^{*}(|-|) \stackrel{\simeq}{\rightarrow} \widehat{C}^{*}$.

(B) $\hat{C}^{*}$ satisfies the following strict excision statement:

Let $(K, L)$ be a pair of ordered simplicial complexes, let $K^{\prime} \subset K$ be a subcomplex and set $L^{\prime}=L \cap K$. If $K^{\prime} \cup L=K$ then the inclusion $i:\left(K^{\prime}, L^{\prime}\right) \subset(K, L)$ induces an isomorphism

$$
\widehat{C}^{*}(i): \widehat{C}^{*}(K, L) \cong \widehat{C}^{*}\left(K^{\prime}, L^{\prime}\right)
$$

where $\widehat{C}^{*}$ is extended to pairs by setting $\widehat{C}^{*}(K, L)=\operatorname{ker}\left(\widehat{C}^{*}(K) \rightarrow \widehat{C}^{*}(L)\right)$.

We treat separately the cases $S^{*}$ and $A_{\mathrm{PL}}$. Suppose first that $C^{*}=S^{*}$ are the singular cochains. Let sSets be the category of simplicial sets. To an ordered simplicial complex $K$, one associates a simplicial set $K_{\bullet}$ defined by

$$
K_{n}=\left\{\left(v_{0}, \ldots, v_{n}\right):\left\{v_{0}, \ldots, v_{n}\right\} \text { is a simplex of } K \text { and } v_{0} \leq \cdots \leq v_{n}\right\}
$$

and the faces and degeneracies are defined by forgetting or repeating a vertex; see Curtis [4, Example (1.3)] or Weibel [17, Example 8.1.8]. We have a homeomorphism $|K| \cong\left|K_{\bullet}\right|$; see [17, Exercise 8.1.4]. 
The normalized chain complex of the free simplicial abelian group $\mathbb{Z}\left[K_{\bullet}\right]$ generated by $K_{\bullet}$ is isomorphic to the oriented chain complex of $K$ as defined in [16, Section 5]. We denote it by $N_{*}\left(\mathbb{Z}\left[K_{\bullet}\right]\right)$ and we consider the dual cochain complex

$$
\widehat{C}^{*}(K):=\operatorname{Hom}\left(N_{*}\left(\mathbb{Z}\left[K_{\bullet}\right]\right), \mathbf{k}\right)
$$

which becomes a k-DGA by defining a cup product through the usual front face/back face formula as in [16, Section 49]. This defines a functor

$$
\widehat{C}^{*}: \mathcal{K} \rightarrow \mathbf{k}-\mathrm{DGA}
$$

and by [16, Theorem 49.1] we have a natural quasi-isomorphism of DGA

$$
C^{*}(|K|)=S^{*}(|K| ; \mathbf{k}) \stackrel{\simeq}{\rightrightarrows} \widehat{C}^{*}(K) .
$$

We check that $\widehat{C}^{*}$ satisfies the strict excision statement. Notice that an element $\phi \in \widehat{C}^{*}(K, L)$ is determined by its values in $\mathbf{k}$ on the simplices of $K$. Suppose that $i:\left(K^{\prime}, L^{\prime}\right) \hookrightarrow(K, L)$ is an inclusion of pairs of ordered simplicial complexes with $L^{\prime}=K^{\prime} \cap L$ and $K=K^{\prime} \cup L$. We show that $\widehat{C}^{*}(i)$ is surjective. Let $\phi^{\prime} \in \widehat{C}^{*}\left(K^{\prime}, L^{\prime}\right)$. Define $\phi \in \widehat{C}^{*}(K, L)$ by $\phi(\sigma)=\phi^{\prime}(\sigma)$ if $\sigma$ is a simplex in $K^{\prime}$ and $\phi(\sigma)=0$ if $\sigma$ is a simplex in $L$. This defines $\phi$ coherently, since $\phi^{\prime}(\sigma)=0$ when $\sigma \in K^{\prime} \cap L=L^{\prime}$, and exhaustively because $K=K^{\prime} \cup L$. Clearly $\widehat{C}^{*}(i)(\phi)=\phi^{\prime}$, hence $\widehat{C}^{*}(i)$ is surjective. For the injectivity notice that if $\phi \in \widehat{C}^{*}(K, L)$ is zero on each simplex of $K^{\prime}$ then it is zero everywhere since it is zero on $L$ and $K=K^{\prime} \cup L$. This proves that $\widehat{C}^{*}$ satisfies condition (B).

Suppose now that $C^{*}=A_{\mathrm{PL}}$ is the functor of piecewise polynomial forms and let $S_{\bullet}(X)$ be the simplicial set of singular simplices of a topological space $X$. Recall from [5, Section 10(c)] that $A_{\mathrm{PL}}$ : Top $\rightarrow$ CDGA actually factors through the functor $S_{\bullet}:$ Top $\rightarrow$ sSets by the way of another functor $A_{\text {PL }}$ sSets $\rightarrow$ CDGA. We define

$$
\widehat{C}^{*}: \mathcal{K} \rightarrow \mathrm{CDGA}, K \mapsto A_{\mathrm{PL}}\left(K_{\bullet}\right) .
$$

For any ordered simplicial complex $K$, the natural weak equivalence $K_{\bullet} \stackrel{\simeq}{\rightarrow} S_{\bullet}\left(\left|K_{\bullet}\right|\right)$ induces a quasi-isomorphism of CDGA

$$
C^{*}(|K|)=A_{\mathrm{PL}}(|K|) \stackrel{\text { def }}{=} A_{\mathrm{PL}}\left(S_{\bullet}(|K|)\right) \cong A_{\mathrm{PL}}\left(S_{\bullet}\left(\left|K_{\bullet}\right|\right)\right) \stackrel{\cong}{\rightarrow} A_{\mathrm{PL}}\left(K_{\bullet}\right) \stackrel{\text { def }}{=} \widehat{C}^{*}(K) \text {. }
$$

An element $\phi \in \widehat{C}^{*}(K, L)$ is determined by its values in $A_{\mathrm{PL}}^{*}$ on the nondegenerated simplices of $K_{\bullet}$, hence on the genuine simplices of $K$. The proof that $\hat{C}^{*}$ satisfies the strong excision isomorphism is analogous to the case $C^{*}=S^{*}(-; \mathbf{k})$, the only difference is that for the surjectivity one needs to make sure that the constructed cochain $\phi$ commutes with the boundaries and degeneracies, which is straightforward. 


\section{Group actions on DGmodules}

Let $G$ be a discrete group. Except when stated otherwise, we will suppose that any action of a group is on the left and that $G$ acts trivially on $\mathbf{k}$. When $G$ acts on two sets we will assume that it acts on its product through the diagonal action. When $G$ acts on a set equipped with additional algebraic structure, we will assume that this action is such that each map defining this structure is equivariant. In particular the action on a $\mathbf{k}-$ module is linear, the action on a tensor product is diagonal, $g \cdot(v \otimes w)=(g \cdot v) \otimes(g \cdot w)$, the action on an algebra $R$ is multiplicative, $g \cdot\left(r \cdot r^{\prime}\right)=(g \cdot r) \cdot\left(g \cdot r^{\prime}\right)$. If $G$ acts on an algebra, $R$, and on an $R$-module, $M$, then we assume that $g \cdot(r \cdot x)=(g \cdot r) \cdot(g \cdot x)$ for $g \in G, r \in R$, and $x \in M$. When $G$ acts on a graded object we assume that the action preserves the degree and for the action on a differential object $(M, d)$, that the differential is equivariant, $d(g \cdot x)=g \cdot(d x)$. If $G$ acts on a $\mathbf{k}$-module $V$, then the dual action of $G$ on $\# V:=\operatorname{Hom}(V, \mathbf{k})$ is the action defined by the formula, for $\phi \in \# V, v \in V$, and $g \in G$,

$$
\langle g \cdot \phi, v\rangle=\left\langle\phi, g^{-1} \cdot v\right\rangle
$$

To emphasize these assumptions, if $R$ is a $\mathbf{k}$-DGA on which $G$ is acting as above, we will say that $R$ is a $G-\mathbf{k}-$ DGA. Also if $M$ is an $R$-DGmodule on which $G$ is acting then we will say that $M$ is a $G-R-D G m o d u l e$.

If $f: M \rightarrow N$ is a morphism of $G-R$-DGmodule, iean equivariant map of $R-$ DGmodules, then its mapping cone $C f=N \oplus s M$ inherits a structure of $G-R-$ DGmodule by $g \cdot(y, s x)=(g \cdot y, s(g \cdot x))$ for $x \in M$ and $y \in N$.

Let $W$ be a topological space equipped with a left continuous action of the group $G$ and recall from Section 4 the functor $C^{*}$ which is either $S^{*}(-; \mathbf{k})$ or $A_{\mathrm{PL}}$. As we noticed at the beginning of that section, an element $\omega \in C^{*}(W)$ is completely determined by its values $\langle\omega, \sigma\rangle$ (in $\mathbf{k}$ or in $A_{\mathrm{PL}}^{*}$ ) on simplices $\sigma \in S_{\bullet}(W)$. We define an action of $G$ on $C^{*}(W)$ by

$$
\langle(g \cdot \omega), \sigma\rangle=\left\langle\omega,\left(g^{-1} \cdot \sigma\right)\right\rangle \quad \text { for } \omega \in C^{*}(W), \sigma \in S_{\bullet}(W), g \in G .
$$

Since the action of $G$ on $S_{\bullet}(W)$ commutes with taking front face and back face, one checks that this induces a structure of $G-\mathbf{k}-\mathrm{DGA}$ on $S^{*}(X ; \mathbf{k})$. It is also straightforward to check that it endows $A_{\mathrm{PL}}(W)$ with a structure of $G-\mathrm{CDGA}$.

If $X \subset W$ is a subspace stable by $G$ (that is, $g \cdot X \subset X$ for $g \in G)$ then $C^{*}(X)$ is also a $G-\mathbf{k}-(\mathrm{C})$ DGA and the restriction map $C^{*}(W) \rightarrow C^{*}(X)$, which is $G$-equivariant and of DGA, endows $C^{*}(X)$ with a structure of $G-C^{*}(W)$-DGmodule.

Suppose that $W$ is the realization of an ordered simplicial complex, also denoted by $W$, and that the action of $G$ respects that triangulation, ie is simplicial and preserves the 
order of vertices. Formulas (5-1) for simplices $\sigma$ of the simplicial complex $W$, defines a structure of $G-\mathbf{k}-(\mathrm{C})$ DGA on the chain complex $\widehat{C}^{*}(W)$ defined in Section 4. Also if $X$ is a subpolyhedron of $W$ stable by the action of $G$ then $\widehat{C}^{*}(X)$ becomes a $G-\widehat{C}^{*}(W)-$ DGmodule.

\section{Orientation twisted action and equivariant Lefschetz dual- ity}

Let $W$ be a closed oriented connected manifold of dimension $n$ on which $G$ acts continuously. We have an induced action on the top homology group $H_{n}(W ; \mathbb{Z})$. This determines a 1-dimensional representation over $\mathbf{k}$ through the homomorphism

$$
\rho: G \rightarrow \mathbb{Z} / 2=\{+1,-1\} \subset \mathbf{k}, g \mapsto \rho(g)
$$

defined by the formula

$$
g \cdot[W]=\rho(g) \cdot[W]
$$

where $[W] \in H_{n}(W ; \mathbb{Z})$ is the orientation class. We call this the orientation representation.

Definition 6.1 Let $G$ be a finite group acting continuously on a closed connected oriented manifold $W$ and let $A$ be a $G-R$-DGmodule. The orientation-twisted action of $G$ on $\# A$ is defined by

$$
\langle g \cdot \phi, a\rangle:=\rho(g)\left\langle\phi, g^{-1} \cdot a\right\rangle \quad \text { for } g \in G, \phi \in \# A, a \in A,
$$

where $\rho$ is the orientation representation (6-1).

In particular if $X \subset W$ is a $G$-invariant subspace of $W$ we have an induced action on $C^{*}(W)$ and $C^{*}(X)$, and we can consider the orientation-twisted dual action on $\# C^{*}(W)$ and $\# C^{*}(X)$. The reason for introducing this twisted action is that it is the correct one to make the Poincare and Lefschetz duality quasi-isomorphisms equivariant as we will see in Theorem 6.3. In order to prove that theorem we need the following proposition. Recall the functor $\widehat{C}^{*}$ from Section 4 .

Proposition 6.2 Let $K$ be an ordered simplicial complex whose realization $W:=|K|$ is a closed oriented connected manifold of dimension $n$. Let $G$ be a finite group acting on the left on $K$ and let $\mathbf{k}$ be a field such that $\operatorname{char}(\mathbf{k})$ does not divide $|G|$. Then there

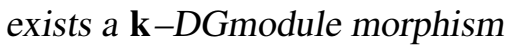

$$
\epsilon_{K}: \widehat{C}^{*}(K) \rightarrow s^{-n} \mathbf{k}
$$

Algebraic $8 \mathcal{G}$ Geometric Topology, Volume 8 (2008) 
such that $\epsilon_{K}^{*}: H^{n}(W) \cong H^{n}\left(s^{-n} \mathbf{k}\right)=\mathbf{k}$ is an isomorphism and such that the $C^{*}(W)-$ DGmodule morphism

$$
\Phi_{K}: \widehat{C}^{*}(K) \stackrel{\simeq}{\rightarrow} s^{-n} \# \widehat{C}^{*}(K),
$$

defined by $\Phi_{K}(\alpha)(\beta)=\epsilon_{K}(\alpha \cdot \beta)$, is $G$-equivariant when $\widehat{C}^{*}(K)$ is equipped with the standard dual action of $G$ and $\# \widehat{C}^{*}(K)$ is equipped with the orientation-twisted $G$-action.

Proof Since $H^{n}(W) \cong \mathbf{k}$ there exists a chain map $\epsilon_{K}^{\prime}: \widehat{C}^{*}(K) \rightarrow s^{-n} \mathbf{k}$ such that $\epsilon_{K}^{\prime}(\mu)=1$ for some cocycle $\mu$ representing the orientation class. Set $\epsilon_{K}(\omega):=$ $(1 /|G|) \sum_{g \in G} \rho(g) \cdot \epsilon_{W}^{\prime}(g \cdot \omega)$. One computes that $\epsilon_{K}(\mu)=1$, so $\epsilon_{K}$ induces an isomorphism in homology. One checks also that $\Phi_{K}$ is $G$-equivariant. The fact that $\Phi_{K}$ is a quasi-isomorphism is a consequence of Poincaré duality.

We arrive to our cochain level equivariant Lefschetz duality theorem:

Theorem 6.3 Let $W$ be an n-dimensional triangulated connected oriented closed manifold. Let $G$ be a finite group that acts simplicially on $W$. Let $f: X \hookrightarrow W$ be a subpolyhedron stable by $G$. Let $\mathbf{k}$ be a field such that char(k) does not divide $|G|$. Let $C^{*}$ be the cochain algebra functor from Section 4. Then there exists a chain of weak equivalences of $G-C^{*}(W)-D G$ modules between $C^{*}(W \backslash X)$ and $s^{-n}\left(\# C^{*}(W) \oplus_{\# C^{*}(f)} s \# C^{*}(X)\right)$, where $\# C^{*}(W)$ and $\# C^{*}(X)$ are equipped with the orientation-twisted dual $G$-action.

Proof As $W$ is triangulated, it is homeomorphic to the realization of an abstract simplicial complex. Replace this simplicial complex by its second barycentric subdivision. The action of $G$ is still simplicial. Moreover there is a natural structure of ordered simplicial complex defined on the barycentric subdivision as follows. Denote by $b(\sigma)$ the barycentre of a simplex $\sigma$ and order the vertices of the barycentric subdivision by $b(\sigma) \leq b(\tau)$ if and only if $\sigma \subset \tau$.

For the rest of the proof, abusing notation, we will denote both the manifold itself and the ordered simplicial complex associated to this second barycentric subdivisions by the same letter $W$, and similarly for other subpolyhedra of $W$.

Let $T$ be the closure of the star of $X$ in the simplicial complex $W$. Since we have took the second subdivision, $T$ is a regular neighborhood of $X$, hence the inclusion $i: X \hookrightarrow$ $T$ is a $G$-equivariant homotopy equivalence. Since $G$ preserves $X$ it also preserves $T$ and the boundary $\partial T$. Denote by $j: T \hookrightarrow W, j_{0}:(T, \partial T) \hookrightarrow(W, \overline{W \backslash T}), i: X \hookrightarrow T$ and $i^{\prime}: \overline{W \backslash T} \hookrightarrow W$ the simplicial $G$-equivariant inclusions. 
Consider the functor $\widehat{C}^{*}$ defined on ordered simplicial complexes in Section 4. By the strict excision property we have an isomorphism

$$
\widehat{C}^{*}\left(j_{0}\right): \widehat{C}^{*}(W, \overline{W \backslash T}) \stackrel{\cong}{\rightrightarrows} \widehat{C}^{*}(T, \partial T) .
$$

Recall the cochain morphism

$$
\epsilon_{W}: \widehat{C}^{*}(W) \stackrel{\simeq}{\rightarrow} s^{-n} \mathbf{k}
$$

from Proposition 6.2 which induces the $G$-equivariant Poincaré duality quasi-isomorphism

$$
\Phi_{W}: \widehat{C}^{*}(W) \stackrel{\simeq}{\rightarrow} s^{-n_{\#}} \widehat{C}^{*}(W), \alpha \mapsto\left(\Phi_{W}(\alpha): \beta \mapsto \epsilon_{W}(\alpha \beta)\right) .
$$

Define $\epsilon_{T}$ as the composite

$$
\epsilon_{T}: \widehat{C}^{*}(T, \partial T) \stackrel{\hat{C}^{*}\left(j_{0}\right)}{\cong} \widehat{C}^{*}(W, \overline{W \backslash T}) \stackrel{\iota}{\rightarrow} \widehat{C}^{*}(W) \stackrel{\epsilon_{W}}{\rightarrow} s^{-n} \mathbf{k} .
$$

This cochain map serves to define a cochain morphism

$$
\Phi_{T}: C^{*}(T, \partial T) \stackrel{\simeq}{\rightarrow} s^{-n} \# C^{*}(T), \psi \mapsto\left(\Phi_{T}(\psi): \tau \mapsto \epsilon_{T}(\psi \tau)\right)
$$

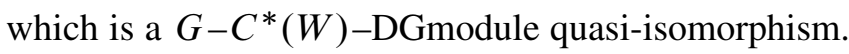

Moreover, since $\epsilon_{T}$ is defined from $\epsilon_{W}$, the following diagram of $G-C^{*}(W)-$ DGmodules commutes:

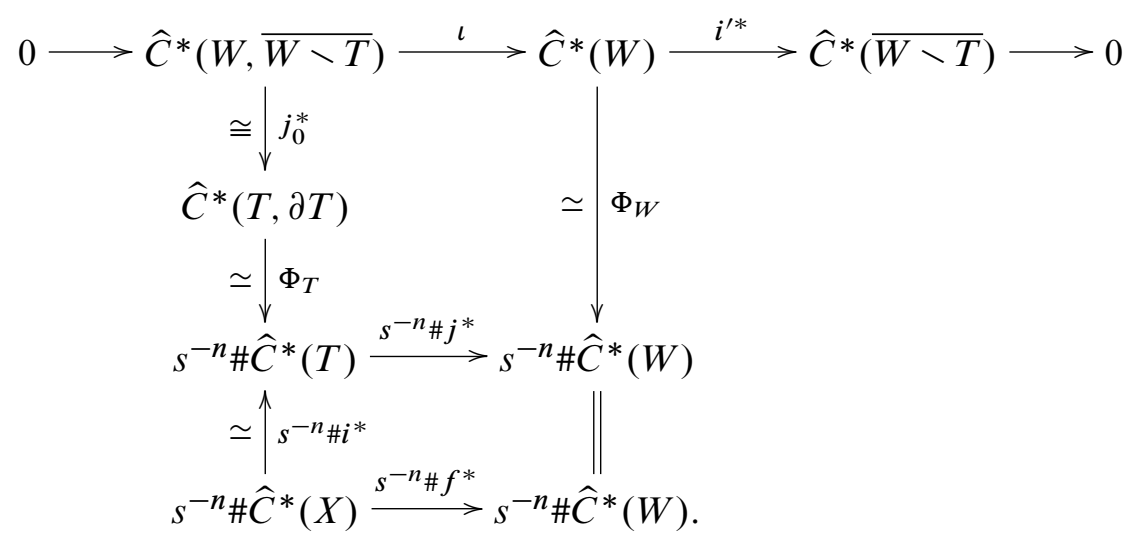

Thus we have the following chain of quasi-isomorphisms of $G-C^{*}(W)$-DGmodules:

$s^{-n}\left(\# \widehat{C}^{*}(W) \oplus_{\# f^{*}} s \# \widehat{C}^{*}(X)\right)$

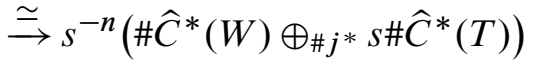

$$
\begin{aligned}
& \stackrel{\simeq}{\longleftarrow} \hat{C}^{*}(W) \oplus_{\iota} \hat{C}^{*}(W, \overline{W \backslash T}) \stackrel{\simeq}{\rightarrow} i^{\prime *} \oplus 0 \hat{C}^{*}(\overline{W \backslash T}) .
\end{aligned}
$$

Algebraic 83 Geometric Topology, Volume 8 (2008) 
The first and last terms of this zigzag of quasi-isomorphisms are respectively

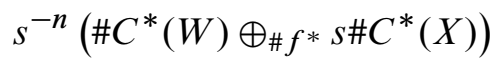

and $C^{*}(\overline{W \backslash T})$. This proves the theorem.

\section{Cubical diagrams and their total cofibres}

Let $E$ be a finite set and let $\Gamma=\left(2^{E}\right)^{\text {op }}$ be the category whose objects are subsets $\gamma$ of $E$ and a morphism $\gamma \rightarrow \gamma^{\prime}$ is a reversed inclusion $\gamma \supset \gamma^{\prime}$. The "shape" of this category is that of an $|E|$-dimensional cube with an initial object $E$ and a final object $\varnothing$.

Definition 7.1 An $E$-cubical diagram in a category $\mathcal{C}$ is a covariant functor

$$
\underline{N}: \Gamma \rightarrow \mathcal{C} .
$$

For $\gamma \subset E$ we denote by $|\gamma|$ the cardinal of that subset. If $e \in \gamma$ we set $\gamma \backslash e:=\gamma \backslash\{e\}$.

Suppose a linear order $\leq$ on $E$ has been given. For $e \in E$ and $\gamma \in \Gamma$ we define the integer

$$
\operatorname{pos}(e: \gamma):=|\{j \in \gamma: j \leq e\}| .
$$

In other words if $\gamma=\left\{e_{1}, \ldots, e_{l}\right\}$ with $e_{1}<\cdots<e_{l}$ then $\operatorname{pos}\left(e_{i}: \gamma\right)=i$.

Definition 7.2 Let $R$ be a $\mathbf{k}$-DGA. Let $E$ be a finite set equipped with a linear ordering. The total cofibre of an $E$-cubical diagram $\underline{N}: \Gamma \rightarrow R$-DGmod of $R-$ DGmodules is the $R$-DGmodule

$$
\operatorname{Tot} \operatorname{Cof}(\underline{N}):=\left(\bigoplus_{\gamma \in \Gamma} y_{\gamma} \cdot \underline{N}(\gamma), D\right)
$$

where, for $\gamma \in \Gamma, x \in \underline{N}(\gamma), r \in R$,

- $y_{\gamma}$ is a variable of degree $-|\gamma|$;

- $\operatorname{deg}\left(y_{\gamma} \cdot x\right)=-|\gamma|+\operatorname{deg}(x)$;

- $r \cdot\left(y_{\gamma} \cdot x\right)=(-1)^{|\gamma| \operatorname{deg}(r)} y_{\gamma} \cdot(r \cdot x)$;

- $D\left(y_{\gamma} \cdot x\right)=(-1)^{|\gamma|} y_{\gamma} \cdot(d(x))+\sum_{e \in \gamma} y_{\gamma \backslash e}(-1)^{\operatorname{pos}(e: \gamma)} \underline{N}(\gamma \rightarrow \gamma \backslash e)(x)$.

The notion of a total cofibre of a cubical diagram was first introduced by Goodwillie in [9]. Actually it is a special case of the following more general definition; see Hüttemann [10]. Let $\Gamma$ be a poset and $\Gamma^{\prime} \subset \Gamma$ be a subposet and let $X: \Gamma \rightarrow \mathcal{C}$ be a 
covariant functor in some Quillen model category. The total cofibre of $X$ is defined as the homotopy cofibre of the map

$$
\operatorname{hocolim}_{\gamma^{\prime} \in \Gamma^{\prime}} X\left(\gamma^{\prime}\right) \rightarrow \text { hocolim }_{\gamma \in \Gamma} X(\gamma) .
$$

In our case $\mathcal{C}$ is the category $R$-DGmod, $\Gamma=\left(2^{E}\right)^{\text {op }}$ and $\Gamma^{\prime}$ is $\Gamma$ without its final object $\varnothing$.

Notice that the definition of the total cofibre depends on the choice of a linear ordering on $E$ but is easy to check that two such linear ordering give isomorphic total cofibres. (Hint: Use Lemma 8.2.)

We introduce the notion of an iterated mapping cone of a bounded chain complex in $R$-DGmod, which extends the usual mapping cone of a chain map. Let

$$
M_{*}:=\left\{M_{r} \stackrel{f_{r}}{\longrightarrow} M_{r-1} \stackrel{f_{r-1}}{\longrightarrow} \cdots \stackrel{f_{2}}{\longrightarrow} M_{1} \stackrel{f_{1}}{\longrightarrow} M_{0}\right\}
$$

where $\left(M_{i}, d_{i}\right)$ and $f_{i}$ are objects and morphisms in $R$-DGmod, for some DGA $R$, such that $f_{i} f_{i+1}=0$. The iterated mapping cone of $M_{*}$ is defined as

$$
C\left(M_{*}\right):=\left(\bigoplus_{i=0}^{r} s^{i} M_{i}, D\right)
$$

with $D\left(s^{i} x\right)=(-1)^{i} s^{i}\left(d_{i} x\right)+s^{i-1} f_{i}(x)$, for $x \in M_{i}$. It is straightforward to check that $D^{2}=0$. When $r=1$ this is the usual mapping cone of the map $f_{1}: M_{1} \rightarrow M_{0}$.

If $\underline{N}: \Gamma \rightarrow$ DGmod is an $E$-cubical diagram of DGmodules with $|E|=r$ then we can define a bounded complex of DGmodules

$$
N_{*}:=\left\{N_{r} \stackrel{f_{r}}{\longrightarrow} N_{r-1} \stackrel{f_{r-1}}{\longrightarrow} \cdots \stackrel{f_{2}}{\longrightarrow} N_{1} \stackrel{f_{1}}{\longrightarrow} N_{0}\right\}
$$

with

$$
N_{i}:=\bigoplus_{\gamma \in \Gamma,|\gamma|=i} \underline{N}(\gamma)
$$

and, for $x \in \underline{N}(\gamma) \subset N_{i}$,

$$
f_{i}(x):=\sum_{e \in \gamma}(-1)^{\operatorname{pos}(e: \gamma)} \underline{N}(\gamma \rightarrow \gamma \backslash e)(x) .
$$

Then it is straightforward to check that $N_{*}$ is a complex of differential modules and that the total cofibre of the cube $\underline{N}$ coincides with the iterated mapping cone of $N_{*}$ :

$$
\operatorname{Tot} \operatorname{Cof}(\underline{N}) \cong C\left(N_{*}\right) .
$$




\section{$8 G$-action on a cubical diagram of $\boldsymbol{R}$-DGmodules}

Let $E$ be a finite set equipped with an action of $G$. This induces a $G$-action on the poset $\Gamma=2^{E}$ that preserves the order (induced by reverse inclusions). Let $\underline{N}: \Gamma \rightarrow$ $R$-DGmod be an $E$-cubical diagram $R$-DGmodules, where $R$ is a $G$-k-DGA.

By a $G$-action on $\underline{N}$ we mean the data of $\mathbf{k}$-linear morphisms

$$
\underline{N}(g, \gamma): \underline{N}(\gamma) \rightarrow \underline{N}(g \cdot \gamma),
$$

for each $g \in G$ and $\gamma \in \Gamma$ such that

- ( $G$-naturality) the following diagrams commute:

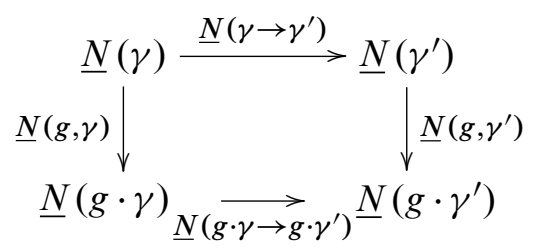

- (associativity) $\underline{N}\left(g^{\prime}, g \cdot \gamma\right) \underline{N}(g, \gamma)=\underline{N}\left(g^{\prime} g, \gamma\right)$;

- (unit) $\underline{N}(1, \gamma)=$ id where $1 \in G$ is the identity;

- $(G-R$-module) for $x \in \underline{N}(\gamma)$ and $r \in R$,

$$
\underline{N}(g, \gamma)(r \cdot x)=(g \cdot r) \cdot(\underline{N}(g, \gamma)(x)) .
$$

For $g \in G, \gamma \in \Gamma$ and $x \in \underline{N}(\gamma)$ we simply write $g \cdot x$ for $\underline{N}(g, \gamma)(x)$ when there is no possible confusion. Then the associativity and unit axioms are the usual axioms $\left(g^{\prime} \cdot g\right) \cdot x=g^{\prime} \cdot(g \cdot x)$ and $1 \cdot x=x$, and the $G-R$-module axioms means that $g \cdot(r \cdot x)=(g \cdot r) \cdot(g \cdot x)$. In particular the maps $\underline{N}(g, \gamma)$ are not maps of $R$-DGmodule.

Notice that if $G$ acts on the $E$-cube $\underline{N}$ then in particular $G$ acts on the $R$-DGmodule $\underline{N}(\varnothing)$.

Suppose given such an $E$-cubical diagram $\underline{N}$ of $R$-DGmodules equipped with a $G$-action as defined above. Fix a linear ordering on $E$. Our goal is to define a $G-$ action on the total cofibre $\operatorname{Tot} \operatorname{Cof}(\underline{N})$ making it a $G-R$-DGmodule. Notice that the "obvious" action $g \cdot\left(y_{\gamma} \cdot x\right)=y_{g \cdot \gamma} \cdot \underline{N}(g, \gamma)(x)$ for $\gamma \in \Gamma, g \in G$ and $x \in \underline{N}(\gamma)$ is not the correct one because it does not make the differential equivariant. 
Definition 8.1 Let $\phi: L \stackrel{\cong}{\Rightarrow} L^{\prime}$ be an bijection between two finite linearly ordered set of cardinality $r \geq 0$, not necessarily order-preserving. We define its signature, $\operatorname{sgn}(\phi)$, as the signature of the permutation in $\Sigma_{r}$ obtained as the composite

$$
\{1, \cdots, r\} \stackrel{\psi}{\rightarrow} L \stackrel{\phi}{\rightarrow} \rightarrow L^{\prime} \stackrel{\psi^{\prime}}{\rightarrow}\{1, \cdots, r\}
$$

where $\psi$ and $\psi^{\prime}$ are the unique order-preserving bijections.

If $E$ is a linearly ordered finite set with an action of a finite group $G$ then for all subset $\gamma \subset E$ and all $g \in G$ the restriction to $\gamma$ gives a bijection $g \mid \gamma: \gamma \stackrel{\cong}{\rightrightarrows} g \cdot \gamma, e \mapsto g \cdot e$, and we denote its signature by $\operatorname{sgn}(g: \gamma)$, where $\gamma$ and $g \cdot \gamma$ are equipped with the linear order induced by $E$.

Lemma 8.2 Let $\gamma \in \Gamma=\left(2^{E}\right)^{\mathrm{op}}$, let $g \in G$ and let $e \in \gamma$. Then

$$
\operatorname{sgn}(g: \gamma) \cdot \operatorname{sgn}(g: \gamma \backslash e)=(-1)^{\operatorname{pos}(e: \gamma)} \cdot(-1)^{\operatorname{pos}(g \cdot e: g \cdot \gamma)}
$$

Proof Straightforward.

Define an action of $G$ on the total cofibre, $\operatorname{Tot} \operatorname{Cof}(\underline{N})$, of Definition 7.2 by

$$
g \cdot y_{\gamma}:=\operatorname{sgn}(g: \gamma) \cdot y_{g \cdot \gamma}
$$

inducing $\quad g \cdot\left(y_{\gamma} \cdot x\right):=\left(g \cdot y_{\gamma}\right) \cdot(g \cdot x)=\operatorname{sgn}(g: \gamma) \cdot y_{g \cdot \gamma} \cdot \underline{N}(g, \gamma)(x)$.

Proposition 8.3 The action defined above induces a $G-R-D G m o d u l e$ structure on $\operatorname{Tot} \operatorname{Cof}(\underline{N})$ such that the inclusion $\underline{N}(\varnothing) \hookrightarrow \operatorname{Tot} \operatorname{Cof}(\underline{N})$ is $G$-equivariant.

Proof Use Lemma 8.2 to prove that the differential is equivariant.

\section{Equivariant Lefschetz theorem for a system of subpolyhe- dra}

Let $W$ be a triangulated space. Let $E$ be a finite set and let

$$
\left\{j_{e}: X_{e} \hookrightarrow W\right\}_{e \in E}
$$

be a collection of subpolyhedra indexed by $e \in E$.

Recall from Section 7 the category $\Gamma=\left(2^{E}\right)^{\mathrm{op}}$. For $\varnothing \neq \gamma \in \Gamma$ set

$$
X_{\gamma}:=\bigcap_{e \in \gamma} X_{e}
$$

and set

$$
X_{\varnothing}:=W \text {. }
$$


This defines a cubical diagram $X_{\bullet}: \Gamma \rightarrow$ Top, $\gamma \mapsto X_{\gamma}$, with the reversed inclusion $\gamma \supset \gamma^{\prime}$ sent to the inclusion $X_{\gamma} \hookrightarrow X_{\gamma^{\prime}}$.

Each $C^{*}\left(X_{\gamma}\right)$ is a right $C^{*}(W)$-DGmodule, therefore its dual $\# C^{*}\left(X_{\gamma}\right)$ is a left $C^{*}(W)$-DGmodule. Moreover if $\gamma \supset \gamma^{\prime}$, the inclusion map $X_{\gamma} \hookrightarrow X_{\gamma^{\prime}}$ induces a morphism

$$
\# C^{*}\left(X_{\gamma}\right) \rightarrow \# C^{*}\left(X_{\gamma^{\prime}}\right) \text {. }
$$

In other words we have an $E$-cubical diagram of $C^{*}(W)$-DGmodules

$$
\# C^{*}\left(X_{\bullet}\right): \Gamma \rightarrow C^{*}(W)-\text { DGmod, } \gamma \mapsto \# C^{*}\left(X_{\gamma}\right) \text {. }
$$

Fix a linear ordering on $E$ and consider the total cofibre of $\# C^{*}\left(X_{\bullet}\right)$. The following is a folklore fact:

Proposition 9.1 With the setting above, if $W=\bigcup_{e \in E} X_{e}$ then the total cofibre $\operatorname{Tot} \operatorname{Cof}\left(\# C^{*}\left(X_{\bullet}\right)\right)$ is acyclic.

Proof When $|E| \leq 1$ the proposition is trivial and for $|E|=2$ it is exactly MayerVietoris theorem. We prove the general case by an induction on the cardinality of $E$. Suppose that the proposition has been proved for $|E| \leq k$ and let $E=E_{0} \cup\{a\}$ with $\left|E_{0}\right|=k$. Set $\Gamma_{0}=\left(2^{E_{0}}\right)^{\mathrm{op}}, W_{0}=\bigcup_{e \in E_{0}} X_{e}, \Gamma=\left(2^{E}\right)^{\mathrm{op}}$, and $W=\bigcup_{e \in E} X_{e}$.

Consider the three systems of subpolyhedra $\left\{X_{e}\right\}_{e \in E_{0}},\left\{X_{e}\right\}_{e \in E}$, and $\left\{X_{a} \cap X_{e}\right\}_{e \in E_{0}}$. The corresponding total cofibres of the associated diagrams, TotCof $\left(\left\{\# C^{*}\left(X_{\gamma}\right)\right\}_{\gamma \in \Gamma_{0}}\right)$, TotCof $\left(\left\{\# C^{*}\left(X_{\gamma}\right)\right\}_{\gamma \in \Gamma}\right)$, and $\operatorname{Tot} \operatorname{Cof}\left(\left\{\# C^{*}\left(X_{a} \cap X_{\gamma}\right)\right\}_{\gamma \in \Gamma_{0}}\right)$, are obtained as the iterated mapping cones of the bounded chain complexes $A_{*}^{\prime}, A_{*}$, and $A_{*}^{\prime \prime}$ defined as follows: For $r \geq 1$, we have

$$
\begin{aligned}
& A_{r}^{\prime}=\bigoplus_{\gamma \in \Gamma_{0},|\gamma|=r} \# C^{*}\left(X_{\gamma}\right), \\
& A_{r}=\bigoplus_{\gamma \in \Gamma,|\gamma|=r} \# C^{*}\left(X_{\gamma}\right), \\
& A_{r}^{\prime \prime}=\bigoplus_{\gamma \in \Gamma_{0},|\gamma|=r} \# C^{*}\left(X_{a} \cap X_{\gamma}\right),
\end{aligned}
$$

and

$$
\begin{aligned}
& A_{0}^{\prime}=\# C^{*}\left(W_{0}\right), \\
& A_{0}=\# C^{*}(W), \\
& A_{0}^{\prime \prime}=\# C^{*}\left(X_{a} \cap W_{0}\right) .
\end{aligned}
$$

For $r \geq 2$ we have obvious short exact sequences

$$
0 \rightarrow A_{r}^{\prime} \rightarrow A_{r} \rightarrow A_{r-1}^{\prime \prime} \rightarrow 0
$$


as well as a short exact sequence

$$
0 \rightarrow A_{1}^{\prime} \rightarrow A_{1} \rightarrow \# C^{*}\left(X_{a}\right) \rightarrow 0 .
$$

Mayer-Vietoris theorem for $W=W_{0} \cup X_{a}$ implies that the commutative square

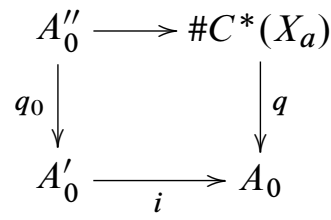

induces a quasi-isomorphism between the mapping cones of the two horizontal arrows of this square

$$
q \oplus s q_{0}: \# C^{*}\left(X_{a}\right) \oplus s A_{0}^{\prime \prime} \stackrel{\cong}{\rightrightarrows} A_{0} \oplus_{i} s A_{0}^{\prime} .
$$

The bulk of the proof is the study of the following commutative diagram of DGmodules:

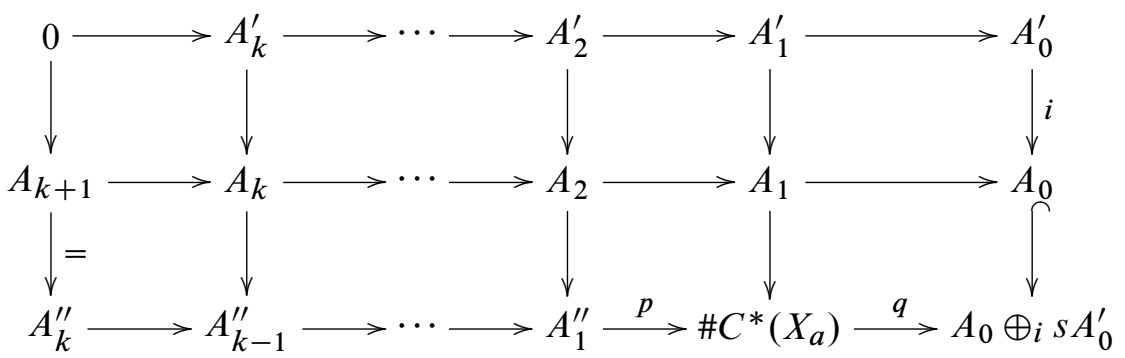

where portions of the horizontal lines are the chain complexes $A_{*}^{\prime}, A_{*}$, and $A_{*}^{\prime \prime}, p$ is the composite of the map $A_{1}^{\prime \prime} \rightarrow A_{0}^{\prime \prime}=\# C^{*}\left(X_{a} \cap W_{0}\right)$ with the map \# $C^{*}\left(X_{a} \cap W_{0}\right) \rightarrow$ $\# C^{*}\left(X_{a}\right)$ induced by the inclusion $X_{a} \cap W_{0} \hookrightarrow X_{a}$, and the vertical arrows are short exact sequences (9-1) and (9-2) except for the rightmost which is the obvious sequence of the mapping cone of $i: A_{0}^{\prime} \rightarrow A_{0}$.

For the sake of the proof we say that a bounded chain complex of DGmodules is quasi-exact if its iterated mapping cone is acyclic. We need to prove that the middle horizontal line of Diagram (9-4) is quasi-exact.

Each vertical sequence in (9-4) is quasi-exact because it is either a short exact sequence or it is the sequence of a mapping cone. The top horizontal line $A_{*}^{\prime}$ is quasi-exact by the induction hypothesis applied to the system $\left\{X_{e}\right\}_{e \in E_{0}}$. We claim that the bottom horizontal line is also quasi-exact. Indeed by induction hypothesis $A_{*}^{\prime \prime}$ is quasiexact. Therefore the iterated mapping cone of the truncated bounded chain complex 
$\left\{A_{k}^{\prime \prime} \rightarrow \cdots \rightarrow A_{1}^{\prime \prime}\right\}$ is quasi-isomorphic to $A_{0}^{\prime \prime}$. By (9-3) we deduce a quasi-isomorphism

$$
\# C^{*}\left(X_{a}\right) \oplus s C\left(\left\{A_{k}^{\prime \prime} \rightarrow \cdots \rightarrow A_{1}^{\prime \prime}\right\}\right) \stackrel{\simeq}{\rightarrow} A_{0} \oplus_{i} s A_{0}^{\prime}
$$

which implies the claim.

We can take the iterated mapping cone of each vertical sequences in Diagram (9-4) and then take the iterated mapping cone of the horizontal chain complexes obtained from these iterated mapping cones. We get an acyclic DGmodule because each term the horizontal complex of iterated mapping cones is acyclic since the vertical sequences are quasi-exact. Working in the opposite order we can first take the iterated mapping cones of each of the three horizontal lines of Diagram (9-4), then take the iterated mapping of the resulting chain complex of these three iterated mapping cones. This iterated mapping cone is also acyclic because the result is independent on the order between the horizontal and vertical directions. Moreover we have proved that the iterated mapping cone of the top and the bottom horizontal lines are acyclic. Therefore the iterated mapping cone of the middle horizontal line is also acyclic.

Suppose that $W$ is an oriented connected closed manifold of dimension $n$. Let $G$ be a finite group acting continuously on $W$. Suppose that $G$ also acts on the set $E$ in such a way that $g \cdot\left(X_{e}\right)=X_{g \cdot e}$ for $g \in G$ and $e \in E$. This induces a $G$-action on the $E$-cubical diagram $\underline{N}:=\# C^{*}\left(X_{\bullet}\right)$ as follows. Recall the orientation representation $\rho$ of (6-1). For $g \in G$ and $\gamma \in \Gamma$ define a morphism

$$
\underline{N}(g, \gamma): \# C^{*}\left(X_{\gamma}\right) \rightarrow \# C^{*}\left(X_{g \cdot \gamma}\right)
$$

as the morphism induced through $\# C^{*}$ by the continuous map $g: X_{\gamma} \rightarrow X_{g \cdot \gamma}$ multiplied by the sign $\rho(g)$. This $G$-action on the cube $\# C^{*}\left(X_{\bullet}\right)$ is called the orientation-twisted action. It is straightforward to check that it defines an action of $E$-cube of $C^{*}(W)$ DGmodules.

Theorem 9.2 Let $W$ be a triangulated oriented connected closed manifold of dimension $n$. Let $E$ be a finite set and let

$$
\left\{j_{e}: X_{e} \hookrightarrow W\right\}_{e \in E}
$$

be a collection of subpolyhedra indexed by $e \in E$. Let $G$ be a finite group acting continuously on the manifold $W$. Suppose that $G$ also acts on the set $E$ in such a way that $g \cdot\left(X_{e}\right)=X_{g \cdot e}$ for $g \in G$ and $e \in E$. Let $\mathbf{k}$ be a field and assume that char(k) does not divide $|G|$. Let $C^{*}$ be the algebra cochain functor of Section 4.

Consider the $E$-cubical diagram of $C^{*}(W)-D G m o d u l e s$

$$
\# C^{*}\left(X_{\bullet}\right): \Gamma=\left(2^{E}\right)^{\mathrm{op}} \rightarrow C^{*}(W)-\mathrm{DGmod}
$$


defined above equipped with the orientation-twisted $G$-action (9-5) and consider the induced action on its total cofibre as in Proposition 8.3.

Then there is a chain of weak equivalences of $G-C^{*}(W)-D G m o d u l e s$ between

(i) $C^{*}\left(W \backslash \bigcup_{e \in E} X_{e}\right)$;

(ii) $s^{-n} \operatorname{Tot} \operatorname{Cof}\left(\# C^{*}\left(X_{\bullet}\right)\right)$.

Proof We first construct a short sequence of $E$-cubical diagrams of $G-C^{*}(W)-$ DGmodules

$$
0 \longrightarrow \underline{N}^{\prime} \stackrel{\mu}{\longrightarrow} \underline{N} \longrightarrow \underline{N}^{\prime \prime} \longrightarrow 0 .
$$

Set $\underline{N}(\gamma):=\# C^{*}\left(X_{\gamma}\right)$ with in particular $\underline{N}(\varnothing)=\# C^{*}(W)$. Define $\underline{N}^{\prime}$ exactly as $\underline{N}$ except that $\underline{N}^{\prime}(\varnothing)=\# C^{*}\left(\bigcup_{e \in E} X_{e}\right)$. The inclusion $f: \bigcup_{e \in E} X_{e} \hookrightarrow W$ induces a map $\mu(\varnothing): \underline{N}^{\prime}(\varnothing) \rightarrow \underline{N}(\varnothing)$ which combined with the identity maps, $\mu(\gamma)=$ id for $\gamma \neq \varnothing$, gives a morphism of $E$-cubical diagram

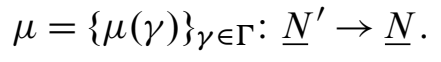

Let $\underline{N}^{\prime \prime}$ be the objectwise mapping cone of $\mu$, that is

$$
\underline{N}^{\prime \prime}:=\left\{\underline{N}(\gamma) \oplus_{\mu}(\gamma) s \underline{N}^{\prime}(\gamma)\right\}_{\gamma \in \Gamma} .
$$

For $\gamma \neq \varnothing, \underline{N}^{\prime \prime}(\gamma)$ is acyclic because it is the mapping cone of the identity map. Therefore the total cofibre of $\underline{N}^{\prime \prime}$ is quasi-isomorphic to

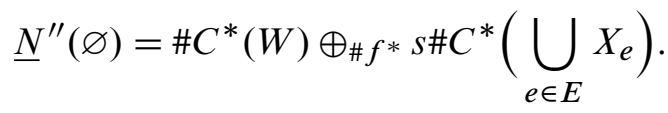

By Theorem 6.3, $C^{*}\left(W \backslash \bigcup_{e \in E} X_{e}\right)$ is weakly equivalent to $s^{-n} \operatorname{Tot} \operatorname{Cof}\left(\underline{N}^{\prime \prime}\right)$, and this weak equivalence is $G$-equivariant by Proposition 8.3.

On the other hand, since $\underline{N}^{\prime \prime}$ is the mapping cone of $\underline{N}^{\prime} \rightarrow \underline{N}$, the short complex of cubes of differential modules (9-6) induces a long exact sequence between the homologies of these cubes. Therefore it also induces a long exact sequence between the homology of their total cofibres. Moreover by Proposition 9.1 the total cofibre of $\underline{N}^{\prime}$ is acyclic, hence we deduce that $s^{-n} \operatorname{Tot} \operatorname{Cof}\left(\underline{N}^{\prime \prime}\right)$ and $s^{-n} \operatorname{Tot} \operatorname{Cof}(\underline{N})$ are weakly equivalent. We have shown above that the first one is weakly equivalent to (i), and the second one is (ii). 


\section{Models for configuration spaces}

We come now to the proof of our main result.

Fix an integer $k \geq 1$ and let $M$ be a closed oriented triangulated manifold of dimension $m$. Consider the action of the symmetric group $\Sigma_{k}$ on $M^{k}$ and on the configuration space $F(M, k)$ by permutation of the factors. Let $A=(A, d)$ be a connected Poincaré duality CDGA weakly equivalent to $A_{\mathrm{PL}}(M)$ and suppose given quasi-isomorphisms of CDGA

$$
A_{\mathrm{PL}}(M) \stackrel{\simeq}{\simeq} \stackrel{\simeq}{\longrightarrow} A
$$

(recall that this exists by the main result of [12].) The inclusion $F(M, k) \hookrightarrow M^{k}$ induces a structure of $\Sigma_{k}-A_{\mathrm{PL}}\left(M^{k}\right)$-DGmodules, hence of $\Sigma_{k}-R^{\otimes k}$-DGmodules, on $A_{\mathrm{PL}}(F(M, k))$. There is also an obvious structure of $\Sigma_{k}-R^{\otimes k}$-DGmodules on the CDGA $F(A, k)$ of Definition 3.4.

Theorem 10.1 With the above setting, there is a weak equivalence of $\Sigma_{k}-R^{\otimes k}$ DGmodules between $A_{\mathrm{PL}}(F(M, k))$ and $F(A, k)$.

The rest of the section is devoted to the proof of that theorem.

The triangulation of $M$ induces a triangulation on the $k$-fold product $W:=M^{k}$ compatible with the action of $\Sigma_{k}$ (Hint: Take the triangulation induced by the prismatic decomposition of $\Delta^{p_{1}} \times \cdots \times \Delta^{p_{k}}$ after fixing a linear order on the vertices of $M$.)

Switching two factors of $M^{k}$ induces a self-map of degree $(-1)^{m}$. Therefore the orientation representation associated to the action of $\Sigma_{k}$ on $M^{k}$ is given by, for $\sigma \in \Sigma_{k}$,

$$
\rho(\sigma)=(\operatorname{sgn}(\sigma))^{m}
$$

Let $E$ be the set

$$
E:=\{(i, j): 1 \leq i<j \leq k\}
$$

linearly ordered by the left lexicographic order. This can be considered as the set of (nonoriented) edges on the set of vertices $\underline{k}=\{1, \cdots, k\}$. Then the objects of $\Gamma=\left(2^{E}\right)^{\text {op }}$ can be interpreted as simple graphs (no loops, no double edges, no orientations on the edges) with vertices in $\underline{k}$.

Suppose given a graph $\gamma \in \Gamma$ and a permutation $\sigma \in \Sigma_{k}$. The lexicographic order on $E$ induces a linear order on $\gamma \subset E$ and $\sigma$ induces a bijection $\sigma: \gamma \stackrel{\cong}{\Rightarrow} \sigma \cdot \gamma$. We can consider its signature $\operatorname{sgn}(\sigma: \gamma)$ as in Definition 8.1, not to be confused with $\operatorname{sgn}(\sigma)$. We denote by $\pi_{0}(\gamma)$ the set of connected components of the graph $\gamma$. In other words 
$\pi_{0}(\gamma)=\{\gamma(1), \cdots, \gamma(r)\}$ is a partition of $\underline{k}$ such that two vertices $i$ and $j$ belongs to the same set $\gamma(u)$ if and only if they are connected by a path in $\gamma$. We define a linear order on $\pi_{0}(\gamma)$ by

$$
\gamma(u) \leq \gamma(v) \Longleftrightarrow \min \gamma(u) \leq \min \gamma(v) .
$$

We denote by $\|\gamma\|:=\left|\pi_{0}(\gamma)\right|$ the number of path components of $\gamma$, not to be confused with the number $|\gamma|$ of edges in $\gamma$. A permutation $\sigma \in \Sigma_{k}$ also induces a bijection $\sigma: \pi_{0}(\gamma) \stackrel{\cong}{\rightrightarrows} \pi_{0}(\sigma \cdot \gamma)$ of ordered sets and we denote its signature by $\operatorname{sgn}\left(\sigma: \pi_{0}(\gamma)\right)$.

For $(i, j) \in E$ set

$$
X_{(i, j)}:=\left\{\left(x_{1}, \cdots, x_{k}\right) \in M^{k} \mid x_{i}=x_{j}\right\} \subset W=M^{k}
$$

which defines a system of subpolyhedra $\left\{X_{e} \subset M^{k}\right\}_{e \in E}$. The associated cubical diagram as in Section 9 is the functor

$$
\left.\underline{\Delta}: \Gamma \rightarrow \text { Top, } \gamma \rightarrow \underline{\Delta}(\gamma)=\left\{x_{1}, \cdots, x_{k}\right) \in M^{k} \mid x_{i}=x_{j} \text { if }(i, j) \in \gamma\right\}=\bigcap_{e \in \gamma} X_{e} .
$$

where the morphisms $\underline{\Delta}\left(\gamma \rightarrow \gamma^{\prime}\right)$ are the obvious inclusions $\underline{\Delta}(\gamma) \hookrightarrow \Delta\left(\gamma^{\prime}\right)$.

We define another functor, $\underline{M}$, naturally homeomorphic to $\underline{\Delta}$. For $\gamma \in \Gamma$, set $\pi_{0}(\gamma)=$ $\{\gamma(1), \cdots, \gamma(r)\}$ with $\gamma(u)<\gamma(v)$ if $1 \leq u<v \leq r=\|\gamma\|$. Set $\underline{M}(\gamma)=M^{\times\|\gamma\|}$, the $r$-fold product of $M$. We have a homeomorphism

$$
h(\gamma): \underline{M}(\gamma) \rightarrow \underline{\Delta}(\gamma),\left(y_{1}, \cdots, y_{r}\right) \rightarrow\left(x_{1}, \cdots, x_{k}\right)
$$

defined by $x_{i}=y_{u}$ if $i \in \gamma(u)$. It is easy to make $\underline{M}$ into a functor such that the homeomorphism $h: \underline{M} \cong \underline{\Delta}$ is natural.

By an iterated diagonal we mean a diagonal map $M \rightarrow M^{p}, x \mapsto(x, \cdots, x)$, for $p \geq 0$. Each map

$$
\underline{M}\left(\gamma \rightarrow \gamma^{\prime}\right): M^{\times\|\gamma\|} \rightarrow M^{\times\left\|\gamma^{\prime}\right\|}
$$

is the composite of a product of $\|\gamma\|$ iterated diagonals followed by a permutation of the $\left\|\gamma^{\prime}\right\|$ factors. These are uniquely determined by $h(\gamma)$.

Recall the Kunneth quasi-isomorphism

$$
A_{\mathrm{PL}}(M)^{\otimes r} \stackrel{\cong}{\rightrightarrows} A_{\mathrm{PL}}\left(M^{\times r}\right), a_{1} \otimes \cdots \otimes a_{r} \mapsto \operatorname{pr}_{1}^{*}\left(a_{1}\right) \cdot \ldots \cdot \operatorname{pr}_{r}^{*}\left(a_{r}\right) .
$$

Through this Kunneth quasi-isomorphism, diagonal maps correspond exactly to the multiplication, and permutations of the factors of $M^{\times r}$ correspond to permutations (with a Koszul sign) of $A_{\mathrm{PL}}(M)^{\otimes r}$. Therefore for each $\gamma \leq \gamma^{\prime} \in \Gamma$ there exists a morphism, obtained as a graded signed permutation followed by iterated multiplications,

$$
\mu\left(\gamma \rightarrow \gamma^{\prime}\right): A_{\mathrm{PL}}(M)^{\otimes\left\|\gamma^{\prime}\right\|} \rightarrow A_{\mathrm{PL}}(M)^{\otimes\|\gamma\|}
$$


making the following diagram commute:

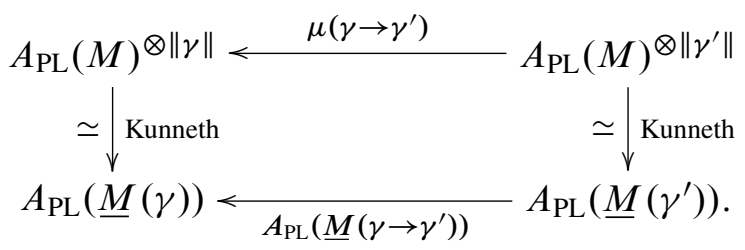

In other words we have build a contravariant functor

$$
\Gamma \rightarrow \mathrm{CDGA}, \gamma \mapsto A_{\mathrm{PL}}(M)^{\otimes\|\gamma\|}
$$

with the morphisms $\mu\left(\gamma \rightarrow \gamma^{\prime}\right)$ obtained as a permutation followed by iterated multiplication and it is naturally quasi-isomorphic to the contravariant functor $A_{\mathrm{PL}}(\underline{M}): \Gamma \rightarrow$ CDGA. Using the quasi-isomorphisms

$$
A_{\mathrm{PL}}(M) \stackrel{\simeq}{\longleftarrow} \stackrel{\simeq}{\longrightarrow} A
$$

and considering similar composite of permutations and iterated multiplications on iterated tensor products of $A$ or $R$, we can build a contravariant functor

$$
\underline{A}: \Gamma \rightarrow \mathrm{CDGA}, \gamma \mapsto \underline{A}(\gamma)=A^{\otimes\|\gamma\|}
$$

naturally weakly equivalent to $A_{\mathrm{PL}}(\underline{M})$.

The duals of the above diagrams give $E$-cubical diagrams of $R^{\otimes k}$-DGmodules. We equip them with the orientation-twisted dual of the action of $\Sigma_{k}$ on the $k$-fold tensor product by permutation of the factors with a Koszul sign. By Theorem 9.2 the $(\mathrm{km})$-th suspension of the total cofibre of the cubical diagram \# $A_{\mathrm{PL}}(\underline{\Delta})$ is equivariantly weakly equivalent to

$$
A_{\mathrm{PL}}\left(M^{k} \backslash \bigcup_{(i, j) \in E} X_{(i, j)}\right)=A_{\mathrm{PL}}(F(M, k)),
$$

as $\Sigma_{k}-R^{\otimes k}$-DGmodules. Therefore $A_{\mathrm{PL}}(F(M, k))$ is quasi-isomorphic as $\Sigma_{k}-$ $R^{\otimes k}$-DGmodules to

$$
s^{-m k} \operatorname{Tot} \operatorname{Cof}(\# \underline{A})=s^{-m k}\left(\bigoplus_{\gamma \in \Gamma} y_{\gamma} \cdot \#\left(A^{\otimes\|\gamma\|}\right)\right) .
$$

To finish the proof of Theorem 10.1 we will build a quasi-isomorphism of $\Sigma_{k}-R^{\otimes k}$ DGmodules

$$
\Phi: s^{-m k} \operatorname{Tot} \operatorname{Cof}(\# \underline{A}) \rightarrow F(A, k) .
$$

This is the content of the following series of lemma. 
First we introduce some notation and terminology.

We say that a graph $\gamma \in \Gamma$ is redundant if there exists an edge $e \in \gamma$ such that $\|\gamma \backslash e\|=\|\gamma\|$.

For an edge $e=(i, j) \in E$ with $1 \leq i<j \leq k$ we set $\Delta(e):=\pi_{i j}^{*}(\Delta) \in A^{\otimes k}$ where $\Delta \in A \otimes A$ is the diagonal class of Equation (3-1).

For a graph $\gamma \in \Gamma$, we set

$$
g_{\gamma}:=\prod_{e \in \gamma} g_{e} \in F(A, k)
$$

where the product is taken in the lexicographic order of $\gamma \subset E$ and $g_{e}=g_{i j}$ is the $(m-1)$-dimensional generator of $F(A, k)$. Notice that if $\gamma$ is a redundant graph then $g_{\gamma}=0$ because of the Arnold relations and $\left(g_{i j}\right)^{2}=0$.

For the Poincare duality algebra $A$ with orientation form $\omega \in \# A^{m}$ we denote by $[A] \in A^{m}$ its fundamental class characterized by $\omega([A])=1$. We have a unique degree $-m$ isomorphism of $A$-module $\theta: A \cong \# A$ characterized by $\theta(1)=\omega$. For $r \geq 1$ we denote by $\epsilon_{r} \in \#\left(A^{\otimes r}\right)^{r m}$ the linear form characterized by $\epsilon_{r}([A] \otimes \cdots \otimes[A])=1$.

The multiplication of the algebra $A$ is denoted by mult: $A \otimes A \rightarrow A$.

Our first three lemmas aim to give an explicit formula for the differential $D$ in the total cofibre (10-1).

Lemma 10.2 (\# mult) $(\theta(1))= \pm(\theta \otimes \theta)(\Delta) \in \#(A \otimes A)$.

Proof Evaluate both sides on the basis $\left\{a_{\lambda} \otimes a_{\mu}^{*}\right\}$.

Lemma 10.3 Let $\gamma \in \Gamma, e \in \gamma$ and set $r=\|\gamma\|$. Then there exist signs $v(\gamma, e) \in$ $\{-1,+1\}$ such that

$$
(\# \underline{A}(\gamma \rightarrow \gamma \backslash e))\left(\epsilon_{r}\right)= \begin{cases}v(\gamma, e) \Delta(e) \cdot \epsilon_{r+1} & \text { if }\|\gamma \backslash e\|>\|\gamma\|, \\ \epsilon_{r} & \text { otherwise. }\end{cases}
$$

Proof In the first case this map is the dual of a signed permutation of $A^{\otimes r+1}$ followed by a multiplication of two adjacent factors. An argument analogous to that of Lemma 10.2 by evaluation on a basis of $A^{\otimes r+1}$ implies the formula.

In the second case, $\|\gamma \backslash e\|=\|\gamma\|$ and $\underline{A}(\gamma \rightarrow \gamma \backslash e)$ is the identity map. 
Lemma 10.4 Let $\gamma \in \Gamma$ be a nonredundant graph. Then the differential $D$ in the total cofibre (10-1) satisfies

$$
D\left(s^{-m k} y_{\gamma} \epsilon\|\gamma\|\right)=(-1)^{m k} \sum_{e \in \gamma} s^{-m k}(-1)^{\operatorname{pos}(e: \gamma)} y_{\gamma \backslash e} \Delta(e) \epsilon_{\|\gamma\|+1} .
$$

Proof Use the formula of $D$ in Definition 7.2 and Lemma 10.3.

The following lemma serves to define signs $\lambda(\gamma)= \pm 1$ that appears in the definition of $\Phi$ in Lemma 10.6. The formula below is exactly the one needed to make $\Phi$ commute with the differential (see Lemma 10.10.)

Lemma 10.5 There exists a map $\lambda: \Gamma \rightarrow\{-1,+1\}$ such that $\lambda(\varnothing)=1$ and for each nonredundant graph $\gamma \in \Gamma$ and $e \in \gamma$

$$
\lambda(\gamma)=-(-1)^{m(\operatorname{pos}(e: \gamma)+\|\gamma\|)} v(\gamma, e) \lambda(\gamma \backslash e) .
$$

Proof Set $R(\gamma, e):=-(-1)^{m(\operatorname{pos}(e: \gamma)+\|\gamma\|)} v(\gamma, e)$ so that the equation of the statement is $\lambda(\gamma)=R(\gamma, e) \cdot \lambda(\gamma \backslash e)$. For a nonredundant graph $\gamma$ we define $\lambda(\gamma)$ by induction on $|\gamma|$ using this equation but we need to prove that it is independent of the choice of the edge $e \in \gamma$. For this it is enough to show that if $e_{1}$ and $e_{2}$ are two distinct edges in $\gamma$ then

$$
R\left(\gamma, e_{1}\right) \cdot R\left(\gamma \backslash e_{1}, e_{2}\right)=R\left(\gamma, e_{2}\right) \cdot R\left(\gamma \backslash e_{2}, e_{1}\right),
$$

which is equivalent to

$$
v\left(\gamma, e_{1}\right) v\left(\gamma \backslash e_{1}, e_{2}\right)=(-1)^{m} v\left(\gamma, e_{2}\right) v\left(\gamma \backslash e_{2}, e_{1}\right) .
$$

Set $r=\|\gamma\|$. Using Lemma 10.3 we compute

$$
\begin{aligned}
\left(\# \underline{A}\left(\gamma \backslash e_{1} \rightarrow \gamma \backslash\left\{e_{1}, e_{2}\right\}\right)\right) & \left(\left(\# \underline{A}\left(\gamma \rightarrow \gamma \backslash e_{1}\right)\right)\left(\epsilon_{r}\right)\right) \\
& =v\left(\gamma, e_{1}\right) \Delta\left(e_{1}\right)\left(\left(\# A\left(\gamma \backslash e_{1} \rightarrow \gamma \backslash\left\{e_{1}, e_{2}\right\}\right)\right)\left(\epsilon_{r+1}\right)\right) \\
& =v\left(\gamma, e_{1}\right) \Delta\left(e_{1}\right) v\left(\gamma \backslash e_{1}, e_{2}\right) \Delta\left(e_{2}\right) \epsilon_{r+2} .
\end{aligned}
$$

A similar computation gives

$$
\begin{array}{r}
\# \underline{A}\left(\gamma \backslash e_{2} \rightarrow \gamma \backslash\left\{e_{1}, e_{2}\right\}\right)\left(\# \underline{A}\left(\gamma \rightarrow \gamma \backslash e_{2}\right)\left(\epsilon_{r}\right)\right) \\
=v\left(\gamma, e_{2}\right) \Delta\left(e_{2}\right) v\left(\gamma \backslash e_{2}, e_{1}\right) \Delta\left(e_{1}\right) \epsilon_{r+2} .
\end{array}
$$

Since \# $\underline{A}$ is a functor, the last two expressions are equal and this implies Equation $(10-2)$ because $\Delta\left(e_{1}\right) \Delta\left(e_{2}\right)=(-1)^{m} \Delta\left(e_{2}\right) \Delta\left(e_{1}\right)$. 
Lemma 10.6 There exists a unique $A^{\otimes k}$-module map

$$
\Phi: s^{-m k} \operatorname{Tot} \operatorname{Cof}(\# \underline{A}) \rightarrow F(A, k)
$$

such that for $\gamma \in \Gamma$,

$$
\Phi\left(s^{-m k} y_{\gamma} \epsilon_{\|\gamma\|}\right)=\lambda(\gamma) g_{\gamma}
$$

Proof The factor $s^{-m k} y_{\gamma} \# A^{\otimes\|\gamma\|}$ is a free $A^{\otimes\|\gamma\|}$-module generated by $s^{-m k} y_{\gamma} \epsilon\|\gamma\|$. Its $A^{\otimes k}$-module structure is induced by an algebra map $A^{\otimes k} \rightarrow A^{\otimes\|\gamma\|}$ obtained as a permutation followed by iterated multiplications. The fact that $\Phi\left(s^{m k} y_{\gamma} \epsilon_{\|\gamma\|}\right)=$ $\lambda(\gamma) g_{\gamma}$ can be extended to a $A^{\otimes k}$-module map is a consequence of the symmetry relations $\pi_{i}^{*}(a) g_{i j}=\pi_{j}^{*}(a) g_{i j}$ in $F(A, k)$.

Notice that if $\gamma$ is a redundant graph then $\Phi\left(s^{-m k} y_{\gamma} \epsilon_{\|\gamma\|}\right)=0$.

The three next lemmas establish the equivariance of $\Phi$.

Lemma 10.7 Let $\gamma \in \Gamma$ and $\sigma \in \Sigma_{k}$. We have the following equation in the total cofibre (10-1):

$$
\sigma \cdot\left(s^{-m k} y_{\gamma} \cdot \epsilon_{\|\gamma\|}\right)=\operatorname{sgn}(\sigma: \gamma)\left(\operatorname{sgn}(\sigma) \operatorname{sgn}\left(\sigma: \pi_{0}(\gamma)\right)\right)^{m} s^{-m k} y_{\sigma \cdot \gamma} \cdot \epsilon_{\|\sigma \cdot \gamma\|} .
$$

Proof The factor $\operatorname{sgn}(\sigma: \gamma)$ is the sign coming from the action on $y_{\gamma}$ in the cubical diagram as in Equation $(8-1), \operatorname{sgn}(\sigma)^{m}$ is the orientation-twisting, and $\operatorname{sgn}\left(\sigma: \pi_{0}(\gamma)\right)^{m}$ is the Koszul sign of the permutation $A^{\otimes\|\gamma\|} \cong A^{\otimes\|\sigma \cdot \gamma\|}$ on an element of top degree.

For $1 \leq p \leq k-1$ and for an edge $e \in E$ or a graph $\gamma \in \Gamma$ we set

$$
\eta_{e}^{p}:=\left\{\begin{array}{ll}
(-1)^{m} & \text { if } e=(p, p+1), \\
+1 & \text { otherwise. }
\end{array} \quad \eta_{\gamma}^{p}:= \begin{cases}(-1)^{m} & \text { if }(p, p+1) \in \gamma \\
+1 & \text { otherwise }\end{cases}\right.
$$

Lemma 10.8 Let $1 \leq p \leq k-1$, consider the transposition $\tau=(p, p+1) \in \Sigma_{k}$, let $\gamma \in \Gamma$ be a nonredundant graph and let $e \in \gamma$. Then

$$
\begin{aligned}
v(\gamma, e) v(\tau \cdot \gamma, \tau \cdot e) & =\eta_{e}^{p}\left(\operatorname{sgn}\left(\tau: \pi_{0}(\gamma)\right) \operatorname{sgn}\left(\tau: \pi_{0}(\gamma \backslash e)\right)\right)^{m} \\
\lambda(\gamma) \lambda(\tau \cdot \gamma) & =\eta_{\gamma}^{p}\left(-\operatorname{sgn}(\tau: \gamma) \operatorname{sgn}\left(\tau: \pi_{0}(\gamma)\right)\right)^{m} \\
\tau \cdot g_{\gamma} & =\eta_{\gamma}^{p} \operatorname{sgn}(\tau: \gamma)^{m-1} g_{\tau \cdot \gamma} .
\end{aligned}
$$


Proof (10-3) Since the differential $D$ on $s^{-m k} \operatorname{Tot} \operatorname{Cof}(\# \underline{A})$ is equivariant we have

$$
\tau . D\left(s^{-m k} y_{\gamma} \cdot \epsilon_{\|\gamma\|}\right)=D\left(\tau . s^{-m k} y_{\gamma} \cdot \epsilon_{\|\gamma\|}\right) .
$$

Develop both sides of this equation using Lemma 10.4 and Lemma 8.2. The sign $\eta_{e}^{p}$ comes from the fact that $\tau \cdot \Delta((p, p+1))=(-1)^{m} \Delta((p, p+1))$.

(10-4) By induction on the number of edges $|\gamma|$ using Lemmas 10.5 and 8.2 and the previous formula. (Hint: in the induction choose the edge $e \in \gamma$ to be $(p, p+1)$ when it belongs to $\gamma$.)

(10-5) The sign $\eta_{\gamma}^{p}$ comes from $g_{p+1, p}=(-1)^{m} g_{p, p+1}$ and the other sign is the Koszul sign of the rearrangement of the $g_{\boldsymbol{e}}$ which are of degree $m-1$.

Lemma 10.9 $\Phi$ is $\Sigma_{k}$-equivariant.

Proof It is enough to check the equivariance for transpositions $\tau=(p, p+1)$ of adjacent vertices applied to the generators $s^{-m k} y_{\gamma} \cdot \epsilon_{\|\gamma\|}$. If $\gamma$ is nonredundant it is a computation using Lemma 10.7 and Lemma 10.8. If $\gamma$ is redundant then the same is true for $\tau \cdot \gamma$ and the images by $\Phi$ of the corresponding generators are 0 .

Lemma 10.10 $\Phi$ commutes with the differentials.

Proof Since $\Phi$ is an $A^{\otimes k}$-module map between $A^{\otimes k}$-DGmodules, it is enough to check this on the generators $s^{-m k} y_{\gamma} \cdot \epsilon_{\|\gamma\|}$. For a nonredundant graph it is a computation using Lemmas 10.4 and 10.5. To finish the proof we establish the following:

Claim If $\gamma$ is a redundant graph then $\Phi\left(D\left(s^{-m k} y_{\gamma} \epsilon_{\|\gamma\|}\right)\right)=0$.

For the sake of the proof we define an $l$-cycle in a graph $\gamma$ as a subset of edges $\left\{\left(i_{1}, i_{2}\right),\left(i_{2}, i_{3}\right), \ldots,\left(i_{l-1}, i_{l}\right),\left(i_{1}, i_{l}\right)\right\}$. A graph $\gamma$ is redundant if and only if it contains some $l$-cycle, with $l \geq 3$, and then $g_{\gamma}=0$. Notice that when $\gamma$ contains more than one cycle, in other words when $\gamma \backslash e$ is still redundant for any edge $e \in \gamma$, then the claim is obvious. So from now on we suppose that $\gamma$ contains exactly one cycle.

The claim is easy for the graph $\gamma_{123}:=\{(1,2),(1,3),(2,3)\}$ using the Arnold relation in $F(A, k)$ (hint: to compare the different signs $\lambda\left(\gamma_{123} \backslash e\right)$, use (10-4) in Lemma 10.8.) By an induction on the number of edges one deduces the claim for any graph containing $\gamma_{123}$ and no other cycle. By the equivariance of $\Phi$ this implies the result for any graph containing a 3-cycle.

Finally one proves the result for any graph containing an $l$-cycle, for $l \geq 4$ by induction on $l$. Indeed if $\gamma$ contains the $l$-cycle $(1,2), \cdots,(l-1, l),(1, l)$ then consider the graph $\hat{\gamma}:=\gamma \cup\{(1,3)\}$. The terms of $D\left(s^{-m k} y_{\hat{\gamma}} \epsilon_{\|\hat{\gamma}\|}\right)$ contains one term indexed by $\gamma$ and other terms indexed by a graph containing a cycle of length $<l$ or more than one cycle. Using that $D^{2}=0$ and the inductive hypothesis one deduces the claim. 
Lemma 10.11 $\Phi$ is a quasi-isomorphism.

Proof Let $\Gamma_{0} \subset \Gamma$ be the subset consisting of graphs of the form $\left\{\left(i_{1}, j_{1}\right), \ldots,\left(i_{l}, j_{l}\right)\right\}$ with $1 \leq i_{1}<\cdots<i_{l} \leq k$ all distinct and $i_{s}<j_{s} \leq k$ for $s=1, \ldots, l$. Consider the inclusion of chain complexes

$$
\iota: s^{-m k}\left(\bigoplus_{\gamma \in \Gamma_{0}} y_{\gamma} \cdot \#\left(A^{\otimes\|\gamma\|}\right)\right) \hookrightarrow s^{-m k}\left(\bigoplus_{\gamma \in \Gamma} y_{\gamma} \cdot \#\left(A^{\otimes\|\gamma\|}\right)\right) .
$$

An argument completely analogous to that of [7, Proposition 2.4] (passing to the duals) shows that $\iota$ is a quasi-isomorphism. Since $\Phi \iota$ is an isomorphism we deduce that $\Phi$ is a quasi-isomorphism.

\section{More general complements and "all-or-nothing" transver- sality}

In summary the idea that we have applied above is that first we have build a DGmodule model of

$$
C^{*}\left(W-\bigcup_{e \in E} X_{e}\right)
$$

of the form of a total cofibre

$$
s^{-n} \operatorname{Tot} \operatorname{Cof}\left(\gamma \mapsto \# C^{*}\left(X_{\gamma}\right)\right)=s^{-n} \bigoplus_{\gamma \in \Gamma} y_{\gamma} \# C^{*}\left(X_{\gamma}\right) .
$$

This only requires a mixture of Lefschetz duality and a general Mayer-Vietoris principle. The disadvantage of this model, which works for any system of subpolyhedra

$$
\left\{X_{e} \hookrightarrow W\right\}_{e \in E}
$$

is that this model has no clear CDGA structure, partly because there is no such algebra structure on the duals $\# C^{*}\left(X_{\gamma}\right)$.

In the case of the configuration space there was another model which (nonequivariantly at least) is isomorphic to

$$
s^{-n} \bigoplus_{\gamma \in \Gamma_{0}} \prod_{e \in \gamma} g_{e} C^{*}\left(X_{\gamma}\right)
$$

which admits a clearer algebra structure. To build this model we have applied Poincaré duality at the cochain level for each of the submanifold $X_{\gamma}$,

$$
s^{-n} y_{\gamma} \# C^{*}\left(X_{\gamma}\right) \simeq \prod_{e \in \gamma} g_{e} C^{*}\left(X_{\gamma}\right)
$$

Algebraic 83 Geometric Topology, Volume 8 (2008) 
For this to make sense we need first each of the $X_{\gamma}$ to be a closed manifold, but also for all these Poincaré dualities at various formal dimensions to fit together to recover the Lefschetz duality for $C^{*}\left(W \backslash \bigcup_{e \in E} X_{e}\right)$ we needed some sort of transversality. In a sense it is exactly to recover this transversality that we had to restrict to a subset $\Gamma_{0} \subset \Gamma$ defined at the beginning of the proof of Lemma 10.11.

In fact this approach can be applied to more general space than configuration spaces. Actually the main points that we here used is the fact that we had an oriented manifold $W$ together with a system of closed submanifolds $X_{\bullet}:=\left\{X_{e} \hookrightarrow W\right\}_{e \in E}$ such that the families of intersections $\left\{\bigcap_{e \in \gamma} X_{e}\right\}_{\gamma \in \Gamma}$ satisfies a certain "all-or-nothing" transversality condition that we now explain.

In the case where $X_{\bullet}$ is a total transverse system of submanifolds, by which we mean that for any disjoint $\gamma_{1}, \gamma_{2} \subset E$ the submanifolds $\bigcap_{e_{1} \in \gamma_{1}} X_{e_{1}}$ and $\bigcap_{e_{2} \in \gamma_{2}} X_{e_{2}}$ intersects transversally, then using cochain-level Poincaré duality gives another model of $C^{*}\left(W \backslash \bigcup_{e \in E} X_{e}\right)$ of the form

$$
\left(\bigoplus_{\gamma \in \Gamma} g_{\gamma} \cdot C^{*}\left(X_{\gamma}\right), D\right)
$$

where $\operatorname{deg}\left(g_{\gamma}\right)=\operatorname{codim}\left(X_{\gamma}\right)$, and there is a natural CDGA structure on this when we think to $g_{\gamma}$ as $\prod_{e_{\in} \gamma} g_{e}$. In other words in the case of a total transverse system we can take $\Gamma_{0}=\Gamma$.

In the case of the configuration space we have $E=\{(i, j): 1 \leq i<j \leq k\}$ and the family of diagonals $X_{\bullet}:=\left\{X_{i j} \hookrightarrow M^{k}\right\}_{(i, j) \in E}$ is certainly not totally transverse, except when $k \leq 2$. But it has another property which we call all-or-nothing transverse. By this we mean that for any $\gamma_{1}, \gamma_{2} \subset E$ the submanifolds $\bigcap_{e_{1} \in \gamma_{1}} X_{e_{1}}$ and $\bigcap_{e_{2} \in \gamma_{2}} X_{e_{2}}$ either intersects transversally or one of them is included in the other. This is the case of the system of diagonals in $M^{k}$. Using that it is then always possible to find a subset $\Gamma_{0} \subset \Gamma$ such that $C^{*}\left(W \backslash \bigcup_{e \in E} X_{e}\right)$ has a model of the form

$$
\left(\bigoplus_{\gamma \in \Gamma_{0}} u_{\gamma} \cdot C^{*}\left(X_{\gamma}\right), D\right)
$$

where $u_{\gamma}=\operatorname{codim}\left(X_{\gamma}\right)$, and again this comes with a natural CDGA structure on this. Actually the subset $\Gamma_{0} \subset \Gamma$ is characterized by the fact that if $\gamma \in \Gamma_{0}$ and $\gamma^{\prime} \subset \gamma$ then $\gamma^{\prime} \in \Gamma_{0}$ and, for $e \notin \gamma$, we have $\gamma \cup\{e\} \in \Gamma_{0}$ if and only if $X_{\gamma \cup\{e\}}^{\prime} \neq X_{\gamma}$. We do not claim that it is a CDGA model in general, and finding suitable conditions for this to be true is certainly an interesting but difficult problem.

This approach could be useful to the study of other complements, like systems of projective subspaces in $\mathbb{C} P(n)$ but we will not develop this further in this paper. 


\section{References}

[1] L Abrams, Two-dimensional topological quantum field theories and Frobenius algebras, J. Knot Theory Ramifications 5 (1996) 569-587 MR1414088

[2] M Bendersky, S Gitler, The cohomology of certain function spaces, Trans. Amer. Math. Soc. 326 (1991) 423-440 MR1010881

[3] A K Bousfield, V K A M Gugenheim, On PL de Rham theory and rational homotopy type, Mem. Amer. Math. Soc. 8(179) (1976) MR0425956

[4] E B Curtis, Simplicial homotopy theory, Advances in Math. 6 (1971) 107-209 MR0279808

[5] Y Félix, S Halperin, J-C Thomas, Rational homotopy theory, Graduate Texts in Math. 205, Springer, New York (2001) MR1802847

[6] Y Félix, D Tanré, The cohomology algebra of unordered configuration spaces, J. London Math. Soc. (2) 72 (2005) 525-544 MR2156668

[7] Y Félix, J-C Thomas, Configuration spaces and Massey products, Int. Math. Res. Not. (2004) 1685-1702 MR2058024

[8] W Fulton, R MacPherson, A compactification of configuration spaces, Ann. of Math. (2) 139 (1994) 183-225 MR1259368

[9] T G Goodwillie, Calculus. II. Analytic functors, K-Theory 5 (1991/92) 295-332 MR1162445

[10] T Hüttemann, Total cofibres of diagrams of spectra, New York J. Math. 11 (2005) 333-343 MR2154359

[11] I Kříž, On the rational homotopy type of configuration spaces, Ann. of Math. (2) 139 (1994) 227-237 MR1274092

[12] P Lambrechts, D Stanley, Poincaré duality and commutative differential graded algebra, to appear in Ann. Sc. E. N. S. arXiv:math/0701309

[13] P Lambrechts, D Stanley, The rational homotopy type of configuration spaces of two points, Ann. Inst. Fourier (Grenoble) 54 (2004) 1029-1052 MR2111020

[14] P Lambrechts, D Stanley, Algebraic models of Poincaré embeddings, Algebr. Geom. Topol. 5 (2005) 135-182 MR2135550

[15] R Longoni, P Salvatore, Configuration spaces are not homotopy invariant, Topology 44 (2005) 375-380 MR2114713

[16] J R Munkres, Elements of algebraic topology, Addison-Wesley, Menlo Park, CA (1984) MR755006

[17] C A Weibel, An introduction to homological algebra, Cambridge Studies in Advanced Math. 38, Cambridge University Press (1994) MR1269324 
Chercheur qualifié au FNRS, Université Catholique de Louvain Institut Mathématique, Chemin du Cyclotron, 2, B-1348 Louvain-la-Neuve, BELGIUM

University of Regina, Department of Mathematics

College West 307.14, Regina, Saskatchewan, S4S 0A2, Canada

pascal.lambrechts@uclouvain.be, stanley@math.uregina.ca

http://milnor.math.ucl.ac.be/plwiki/PascalLambrechtsProfessional/

HomePage

Received: 17 July 2007 Revised: 19 March 2008

Algebraic 8 Geometric Topology, Volume 8 (2008) 